Article

\title{
A Micromechanical Fatigue Limit Stress Model of Fiber-Reinforced Ceramic-Matrix Composites under Stochastic Overloading Stress
}

\author{
Longbiao Li \\ College of Civil Aviation, Nanjing University of Aeronautics and Astronautics, No. 29 Yudao St., Nanjing 210016, \\ China; 1lb451@nuaa.edu.cn
}

Received: 5 July 2020; Accepted: 23 July 2020; Published: 24 July 2020

check for updates

\begin{abstract}
Fatigue limit stress is a key design parameter for the structure fatigue design of composite materials. In this paper, a micromechanical fatigue limit stress model of fiber-reinforced ceramic-matrix composites (CMCs) subjected to stochastic overloading stress is developed. The fatigue limit stress of different carbon fiber-reinforced silicon carbide (C/SiC) composites (i.e., unidirectional (UD), cross-ply $(\mathrm{CP}), 2 \mathrm{D}, 2.5 \mathrm{D}$, and $3 \mathrm{D} \mathrm{C} / \mathrm{SiC}$ ) is predicted based on the micromechanical fatigue damage models and fatigue failure criterion. Under cyclic fatigue loading, the fatigue damage and fracture under stochastic overloading stress at different applied cycle numbers are characterized using two parameters of fatigue life decreasing rate and broken fiber fraction. The relationships between the fatigue life decreasing rate, stochastic overloading stress level and corresponding occurrence applied cycle number, and broken fiber fraction are analyzed. Under the same stochastic overloading stress level, the fatigue life decreasing rate increases with the occurrence applied cycle of stochastic overloading, and thus, is the highest for the cross-ply C/SiC composite and lowest for the $2.5 \mathrm{D} \mathrm{C} / \mathrm{SiC}$ composite. Among the UD, 2D, and 3D C/SiC composites, at the initial stage of cyclic fatigue loading, under the same stochastic overloading stress, the fatigue life decreasing rate of the $3 \mathrm{D} \mathrm{C/SiC}$ is the highest; however, with the increasing applied cycle number, the fatigue life decreasing rate of the UD $\mathrm{C} / \mathrm{SiC}$ composite is the highest. The broken fiber fraction increases when stochastic overloading stress occurs, and the difference of the broken fiber fraction between the fatigue limit stress and stochastic overloading stress level increases with the occurrence applied cycle.
\end{abstract}

Keywords: fatigue limit stress; ceramic-matrix composites (CMCs); stochastic overloading stress; fiber failure

\section{Introduction}

Ceramic-matrix composites (CMCs) possess high specific strength and specific modulus, high temperature resistance, and have already been applied on hot section components of commercial aero engines [1-3]. To ensure the reliability and safety of CMC components, it is necessary to develop performance evaluation, damage evolution, strength, and life prediction tools for airworthiness certification [4].

Under cyclic fatigue loading, matrix cracking, interface debonding, interface wear, and fiber fracture occur with the applied cycle, and these fatigue damage mechanisms degrade the mechanical performance of fiber-reinforced CMCs [5-7]. Fatigue limit stress is a key parameter for the design of CMC components. However, fatigue limit stress of CMCs depends on many factors, i.e., fiber characteristic and fiber properties [8,9], loading frequency [10,11], temperature [12,13], and testing conditions [14-16]. Under cyclic fatigue loading, stochastic overloading stress may occur due to a special operation condition of the aero engine, which can affect the internal fatigue damage evolution and 
lifetime of CMCs [17,18]. Reynaud [5], Evans [19], and Li [20-22] developed micromechanical fatigue life prediction methods for fiber-reinforced CMCs considering different fatigue damage mechanisms. The degradation rate of the fiber/matrix interface shear stress and fiber strength affects the fatigue life and fatigue limit stress. However, in the developed micromechanical model, the effect of stochastic overloading stress on fatigue limit stress has not been considered.

In this paper, a micromechanical fatigue limit stress model of fiber-reinforced CMCs subjected to stochastic overloading stress is developed. The fatigue limit stress for different carbon fiber-reinforced silicon carbide $(\mathrm{C} / \mathrm{SiC})$ composites is predicted based on the micromechanical fatigue damage models and fatigue failure criterion. The relationships between the fatigue life decreasing rate, stochastic overloading stress level and corresponding occurrence applied cycle number, and broken fiber fraction are analyzed.

\section{Theoretical Model}

When stochastic overloading stress occurs under cyclic fatigue loading, the fatigue damage evolution of matrix cracking, interface debonding, and fiber failure are affected. Figure 1 shows stochastic overloading stress $\sigma_{\mathrm{s}}$ occurred at different applied cycle numbers. In the present analysis, the overloading stress $\sigma_{\mathrm{s}}$ remains the same at applied cycle numbers $N_{1}, N_{2}$, and $N_{3}$.

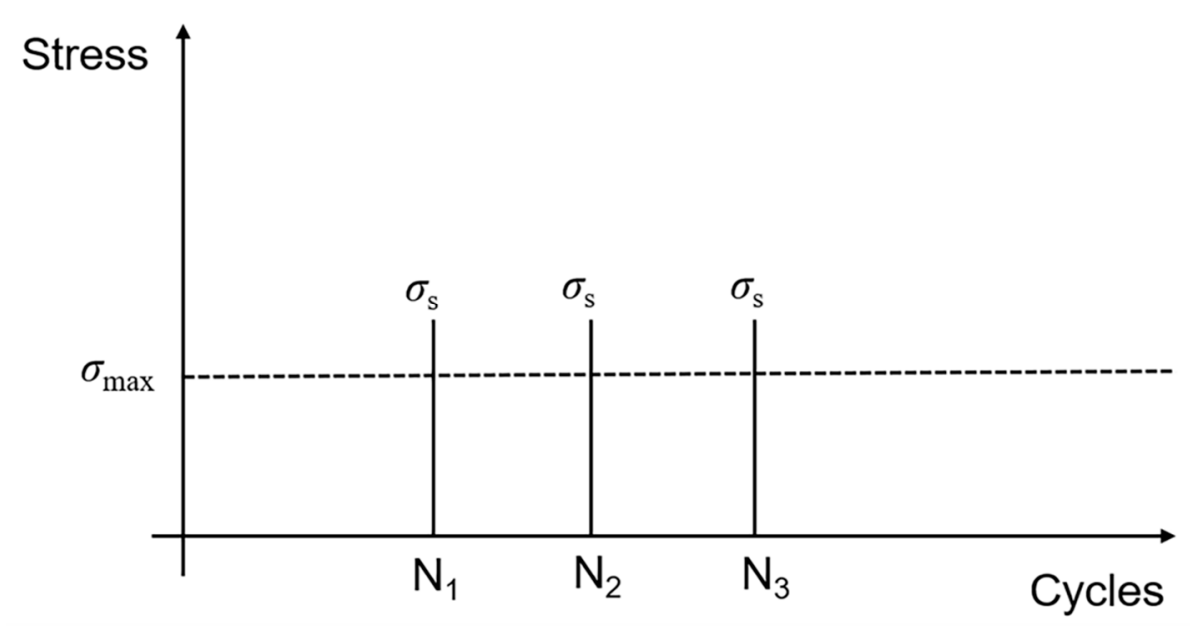

Figure 1. Diagram of stochastic overloading stress under cyclic fatigue loading.

Based on the global load sharing (GLS) criterion, under stochastic overloading stress, the stress carried by intact and broken fiber is determined by Equation (1) [23].

$$
\frac{\sigma_{\mathrm{s}}}{V_{\mathrm{f}}}=\Phi_{\mathrm{s}}\left(1-P_{\mathrm{f}}\right)+\Phi_{\mathrm{b}} P_{\mathrm{f}}
$$

where $V_{\mathrm{f}}$ is the fiber volume, $\Phi_{\mathrm{s}}$ is the intact fiber stress under stochastic overloading stress, $\Phi_{\mathrm{b}}$ is the stress carried by broken fiber, and $P_{\mathrm{f}}$ is the fiber failure probability, and can be determined by Equation (2).

$$
\begin{gathered}
P_{\mathrm{f}}=1-\exp \left[-\Theta \Omega^{m}\left(\frac{\Phi_{\mathrm{s}}}{\sigma_{\mathrm{c}}}\right)^{m+1}\right] \\
\Phi_{\mathrm{b}}=\frac{\Phi_{\mathrm{s}}}{\Theta \Omega^{m} P_{\mathrm{f}}}\left(\frac{\sigma_{\mathrm{c}}}{\Phi_{\mathrm{s}}}\right)^{m+1}\left\{1-\exp \left[-\Theta \Omega^{m}\left(\frac{\Phi_{\mathrm{s}}}{\sigma_{\mathrm{c}}}\right)^{m+1}\right]\right\}-\frac{\Phi_{\mathrm{s}}}{P_{\mathrm{f}}} \exp \left[-\Theta \Omega^{m}\left(\frac{\Phi_{\mathrm{s}}}{\sigma_{\mathrm{c}}}\right)^{m+1}\right]
\end{gathered}
$$

where $m$ is the fiber Weibull modulus, and $\sigma_{\mathrm{c}}$ is the fiber characteristic strength, and $\Theta$ and $\Omega$ denote the degradation rate of the interface shear stress and fiber strength and can be determined by Equations (4) and (5), respectively.

$$
\Theta(N)=\frac{1}{1-(1-\varphi)\left[1-\exp \left(-\omega N^{\lambda}\right)\right]}
$$




$$
\Omega(N)=\frac{1}{1-p_{1}(\log N)^{p_{2}}}
$$

where $\varphi$ is the ratio between the steady interface shear stress and initial interface shear stress, $\omega$ and $\lambda$ are the interface wear model parameter, and $p_{1}$ and $p_{2}$ are the fiber strength degradation model parameter.

Substituting Equations (2) and (3) into Equation (1), the relation between the applied stress and fiber intact stress is determined by Equations (6).

$$
\frac{\sigma_{\mathrm{s}}}{V_{\mathrm{f}}}=\frac{\Phi_{\mathrm{s}}}{\Theta \Omega^{m}}\left(\frac{\sigma_{\mathrm{c}}}{\Phi_{\mathrm{s}}}\right)^{m+1}\left\{1-\exp \left[-\Theta \Omega^{m}\left(\frac{\Phi_{\mathrm{s}}}{\sigma_{\mathrm{c}}}\right)^{m+1}\right]\right\}
$$

Using Equations (4)-(6), the intact fiber stress under stochastic overloading stress can be obtained with the occurrence applied cycle number. Substituting the intact fiber stress under stochastic overloading stress into Equation (2), the fraction of broken fiber under stochastic overloading stress can be obtained. Under cyclic fatigue loading, when the fiber failure probability approaches the critical value, the composite fatigue fractures. The fatigue limit stress of CMCs at room temperature can be obtained using the developed life prediction model and fatigue limit cycle number $N_{\text {limit }}$.

The fatigue life decreasing rate is defined by Equation (7).

$$
\Lambda=\frac{N_{\mathrm{f}}\left(\sigma_{\text {limit }}\right)-N_{\mathrm{f}}\left(\sigma_{\mathrm{s}}\right)}{N_{\mathrm{f}}\left(\sigma_{\text {limit }}\right)}
$$

where $N_{\mathrm{f}}\left(\sigma_{\text {limit }}\right)$ is the fatigue failure cycle number under fatigue limit stress, and $N_{\mathrm{f}}\left(\sigma_{\mathrm{s}}\right)$ is the fatigue failure cycle number under stochastic overloading stress.

\section{Experimental Comparisons}

Under cyclic fatigue loading, stochastic overloading stress affects the fatigue damage evolution, i.e., increasing the fiber failure probability, and decreasing the fatigue life. In this section, the fatigue limit stress of different $\mathrm{C} / \mathrm{SiC}$ composites is predicted. The material properties and fatigue damage model parameters of the $\mathrm{C} / \mathrm{SiC}$ composite are listed in Table 1 . Under fatigue limit stress, stochastic overloading occurring in different applied cycle numbers can decrease the fatigue life. Using the developed fiber failure model in Equation (2) and the fatigue damage models in Equations (4) and (5), the effect of the stochastic overloading stress level and corresponding occurrence cycle number on the fatigue limit stress and corresponding fatigue life is analyzed. The relationships between the stochastic overloading stress level, occurrence cycle number, broken fiber fraction, and fatigue limit stress are established.

\subsection{Unidirectional C/SiC Composite}

The unidirectional $\mathrm{T}-700^{\mathrm{TM}}$ carbon fiber-reinforced silicon carbide composite was fabricated using the hot-pressing (HP) method. Low pressure chemical vapor infiltration was employed to deposit approximately 5-20 layers of PyC/SiC with the mean thickness of $0.2 \mu \mathrm{m}$. The nano-SiC powder and sintering additives were ball milled for $4 \mathrm{~h}$ using $\mathrm{SiC}$ balls. After drying, the powders were dispersed in xylene with polycarbonsilane (PCS) to form the slurry. Carbon fiber tows were infiltrated by the slurry and wound to form aligned unidirectional composite sheets. After drying, the sheets were cut to a size of $150 \mathrm{~mm} \times 150 \mathrm{~mm}$ and pyrolyzed in argon. Then the sheets were stacked in a graphite die and sintered by hot pressing. The dog-bone shaped specimens were cut from $150 \mathrm{~mm} \times 150 \mathrm{~mm}$ panels by water cutting. The tension-tension fatigue tests were conducted on a MTS Model 809 servo hydraulic load-frame (MTS Systems Corp., Minneapolis, MN, USA). The fatigue experiments were in a sinusoidal wave form with a loading frequency $f=10 \mathrm{~Hz}$. The fatigue load ratio $\left(\sigma_{\min } / \sigma_{\max }\right)$ was $R=$ 0.1. The fatigue tests were conducted under load control at room temperature. 
Table 1. Material properties of carbon fiber-reinforced silicon carbide (C/SiC) composite.

\begin{tabular}{|c|c|c|c|c|c|}
\hline Items & Unidirectional [7] & Cross-Ply [13] & 2D [10] & $2.5 \mathrm{D}[8]$ & 3D [9] \\
\hline Manufacturing Process & Hot Pressing & Hot Pressing & $\begin{array}{l}\text { Chemical Vapor } \\
\text { Infiltration (CVI) }\end{array}$ & CVI & CVI \\
\hline Stress Ratio & 0.1 & 0.1 & 0.1 & 0.1 & 0.1 \\
\hline Frequency/(Hz) & 10 & 10 & 10 & 10 & 60 \\
\hline Fiber Type & $\mathrm{T}-700^{\mathrm{TM}}$ & $\mathrm{T}-700^{\mathrm{TM}}$ & $\mathrm{T}-300^{\mathrm{TM}}$ & $\mathrm{T}-300^{\mathrm{TM}}$ & $\mathrm{T}-300^{\mathrm{TM}}$ \\
\hline$V_{\mathrm{f}}$ & 0.4 & 0.4 & 0.45 & 0.4 & 0.4 \\
\hline$\sigma_{\text {uts }}{ }^{1} /(\mathrm{MPa})$ & 270 & 124 & 420 & 225 & 276 \\
\hline$r_{\mathrm{f}}{ }^{2} /(\mu \mathrm{m})$ & 3.5 & 3.5 & 3.5 & 3.5 & 3.5 \\
\hline$\tau_{\text {io }}{ }^{3} /(\mathrm{MPa})$ & 8 & 6.2 & 25 & 20 & 20 \\
\hline$\tau_{\text {imin }}{ }^{4} /(\mathbf{M P a})$ & 0.3 & 1.5 & 8 & 8 & 5 \\
\hline$\omega^{5}$ & 0.04 & 0.06 & 0.002 & 0.001 & 0.02 \\
\hline$\Lambda^{5}$ & 1.5 & 1.8 & 1.0 & 1.0 & 1.0 \\
\hline$p_{1}{ }^{6}$ & 0.01 & 0.01 & 0.018 & 0.02 & 0.012 \\
\hline$p_{2}{ }^{6}$ & 1.0 & 0.8 & 1.0 & 1.2 & 1.0 \\
\hline$m^{7}$ & 5 & 5 & 5 & 5 & 5 \\
\hline
\end{tabular}

\footnotetext{
${ }^{1} \sigma_{\text {uts }}$ is composite tensile strength; ${ }^{2} r_{\mathrm{f}}$ is the fiber radius; ${ }^{3} \tau_{\text {io }}$ is the interface shear stress upon initial loading;

${ }^{4} \tau_{\text {imin }}$ is the steady-state interface shear stress; ${ }^{5} \omega$ and $\lambda$ are the interface degradation model parameters; ${ }^{6} p_{1}$ and
} $p_{2}$ are the fiber strength degradation model parameters; ${ }^{7} \mathrm{~m}$ is the fiber Weibull modulus.

Figure 2 shows the experimental and predicted fatigue life $\mathrm{S}-\mathrm{N}$ curves of the unidirectional $\mathrm{C} / \mathrm{SiC}$ composite. When the fatigue limit applied cycle number is defined to be $N_{\text {limit }}=10^{6}$, the corresponding predicted fatigue limit stress is approximately $\sigma_{\text {limit }}=241 \mathrm{MPa}$ (approximately $89.2 \% \sigma_{\text {uts }}$ ).
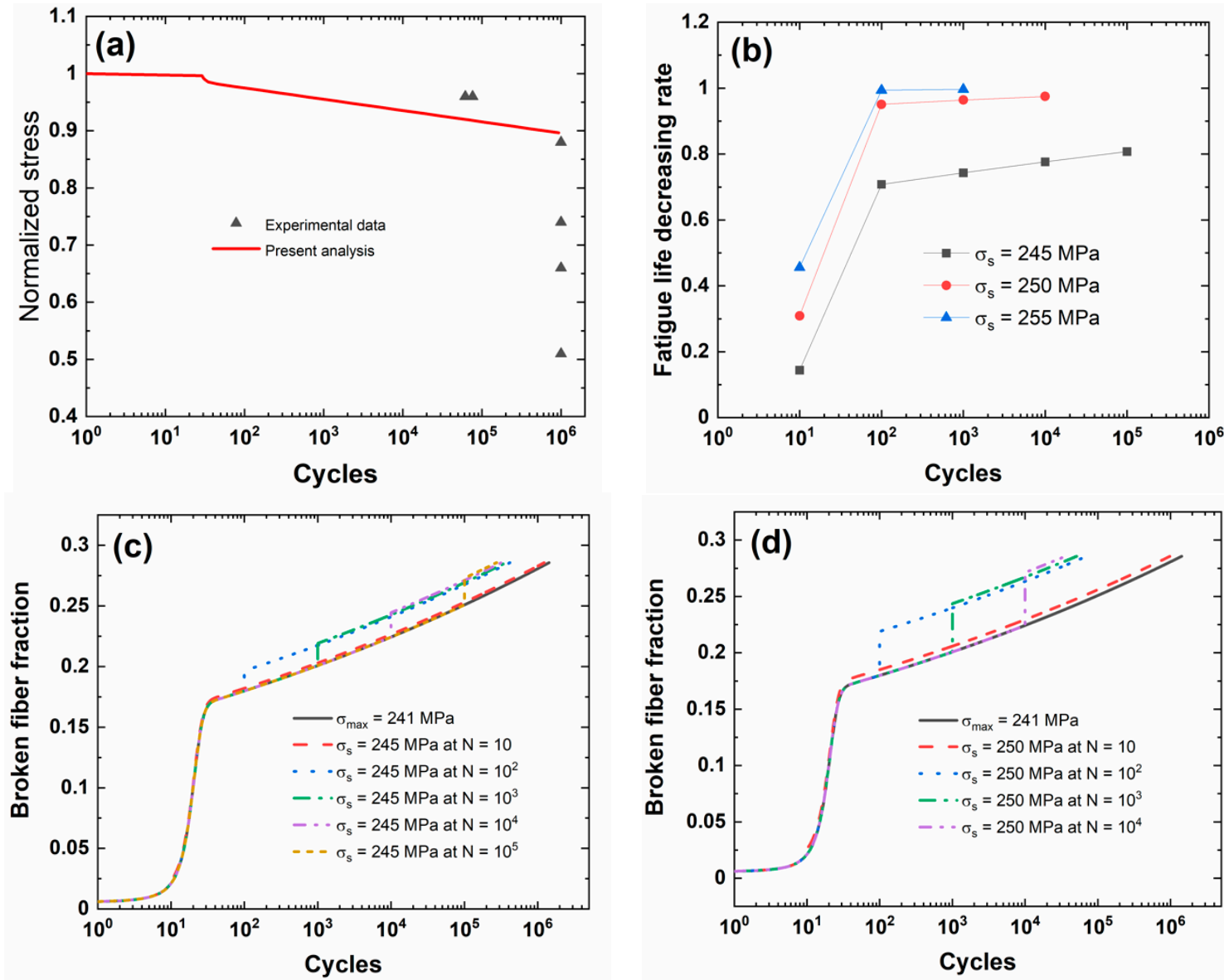

Figure 2. Cont. 


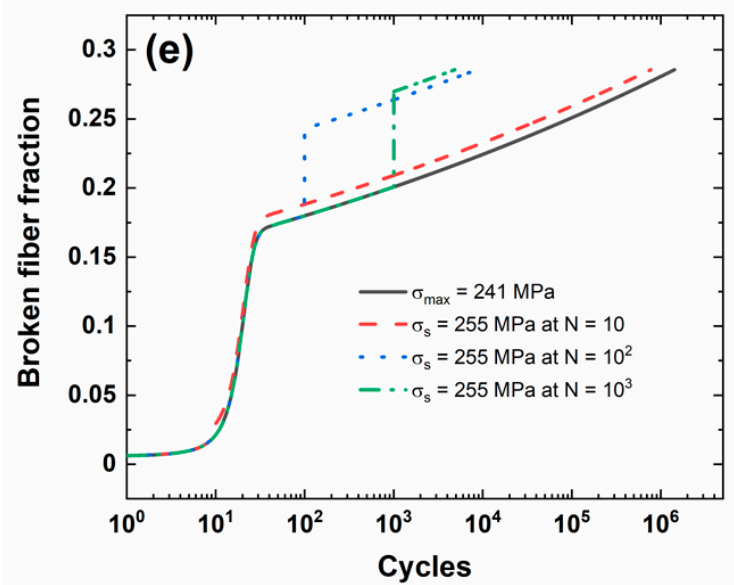

Figure 2. (a) Experimental and predicted fatigue life S-N curves; (b) the fatigue life decreasing rate versus occurrence applied cycle number curve for different stochastic loading stress levels; (c) the broken fiber fraction versus applied cycle number curves under $\sigma_{\text {limit }}=241 \mathrm{MPa}$ and stochastic overloading stress $\sigma_{\mathrm{s}}=245 \mathrm{MPa}$ at $N_{\mathrm{S}}=10,10^{2}, 10^{3}, 10^{4}$, and $10^{5} ;(\mathbf{d})$ the broken fiber fraction versus applied cycle number curves under $\sigma_{\text {limit }}=241 \mathrm{MPa}$ and stochastic overloading stress $\sigma_{\mathrm{s}}=250 \mathrm{MPa}$ at $N_{\mathrm{s}}=10,10^{2}$, $10^{3}, 10^{4}$, and $10^{5}$; and, (e) the broken fiber fraction versus applied cycle number curves under $\sigma_{\text {limit }}$ $=241 \mathrm{MPa}$ and stochastic overloading stress $\sigma_{\mathrm{S}}=255 \mathrm{MPa}$ at $N_{\mathrm{S}}=10,10^{2}$, and $10^{3}$ of unidirectional $\mathrm{C} / \mathrm{SiC}$ composite.

Figure $2 \mathrm{~b}$ shows the fatigue life decreasing rate versus the occurrence cycle number of stochastic overloading curves for different stochastic overloading stress levels of $\sigma_{\mathrm{s}}=245,250$, and $255 \mathrm{MPa}$ (i.e., approximately 1.016, 1.037, 1.058 of fatigue limit stress). During the application of CMC components, the overloading stress level is not high, and the low overloading stress level is chosen for analysis. Under the same stochastic overloading stress level of $\sigma_{\mathrm{s}}=245,250$, and $255 \mathrm{MPa}$, the fatigue life decreasing rate increases with the occurrence applied cycle of stochastic overloading (i.e., $N_{\mathrm{s}}=10,10^{2}$, $10^{3}, 10^{4}$, and $\left.10^{5}\right)$. Under $\sigma_{\mathrm{s}}=245 \mathrm{MPa}$, the fatigue life decreasing rate increases from $\Lambda=0.14392$ at $N_{\mathrm{S}}=10$ to $\Lambda=0.8074$ at $N_{\mathrm{S}}=10^{5} ;$ under $\sigma_{\mathrm{s}}=250 \mathrm{MPa}$, the fatigue life decreasing rate increases from $\Lambda=0.3091$ at $N_{\mathrm{s}}=10$ to $\Lambda=0.97479$ at $N_{\mathrm{s}}=10^{4}$; and under $\sigma_{\mathrm{s}}=255 \mathrm{MPa}$, the fatigue life decreasing rate increases from $\Lambda=0.4559$ at $N_{\mathrm{S}}=10$ to $\Lambda=0.9966$ at $N_{\mathrm{s}}=10^{3}$. When the applied cycle is between $N_{\mathrm{S}}=$ 10 and $10^{2}$, the fatigue life decreasing rate increases rapidly with the occurrence applied cycle; however, when the applied cycle is higher than $N_{\mathrm{s}}=10^{2}$, the fatigue life decreasing rate increases slowly with the applied cycle. At the initial stage of cyclic fatigue loading, the fatigue damage mechanisms of matrix cracking, interface debonding and wear depend on the fatigue peak stress level. The occurrence of stochastic overloading stress at the initial stage of cyclic fatigue loading deteriorates fatigue damage evolution, i.e., decreasing matrix crack spacing, increasing interface debonding length and broken fiber fraction; however, when matrix cracking and interface wear approach a steady-state, the effect of stochastic overloading stress on fatigue damage or fatigue life decreasing rate decreases.

Figure 2c-e shows the broken fiber fraction versus the applied cycle number curves for different stochastic overloading stress levels and occurrence applied cycle numbers. The broken fiber fraction increases when stochastic overloading stress occurs, and the difference of the broken fiber fraction between original peak stress and stochastic overloading stress level increases with the applied cycle number.

Table 2 shows the fatigue limit stress and broken fiber fraction at different occurrence cycle numbers and stochastic overloading stress. When stochastic overloading stress $\sigma_{\mathrm{s}}=245 \mathrm{MPa}$ occurs at applied cycles $N_{\mathrm{s}}=10,10^{2}, 10^{3}, 10^{4}$, and $10^{5}$, the broken fiber fraction increases from $P_{\mathrm{f}}=0.02113$, $0.17991,0.20077,0.22431$, and 0.25085 under $\sigma_{\text {limit }}=241 \mathrm{MPa}$ to $P_{\mathrm{f}}=0.02329,0.19662,0.21915,0.2445$, and 0.27298; when stochastic overloading stress $\sigma_{\mathrm{s}}=250 \mathrm{MPa}$ occurs at applied cycles $N_{\mathrm{s}}=10,10^{2}$, $10^{3}$, and $10^{4}$, the broken fiber fraction increases to $P_{\mathrm{f}}=0.02626,0.21897,0.24365$, and 0.27131 ; finally, 
when stochastic overloading stress $\sigma_{\mathrm{S}}=255 \mathrm{MPa}$ occurs at applied cycles $N=10,10^{2}$, and $10^{3}$, the broken fiber fraction increases to $P_{\mathrm{f}}=0.02952,0.24295$, and 0.26984 .

Table 2. Fatigue limit stress and broken fiber fraction of unidirectional $\mathrm{C} / \mathrm{SiC}$ composite under stochastic overloading stress.

\begin{tabular}{|c|c|c|c|c|c|c|c|c|}
\hline \multirow{2}{*}{$\sigma_{\max }=241 \mathrm{MPa}$} & \multicolumn{2}{|l|}{$N_{\mathrm{f}}{ }^{2}$} & \multirow{2}{*}{$\begin{array}{l}N^{3}=1 \\
0.00609\end{array}$} & \multirow{2}{*}{$\begin{array}{l}N=10 \\
0.02113\end{array}$} & \multirow{2}{*}{$\begin{array}{l}N=10^{2} \\
0.17991\end{array}$} & \multirow{2}{*}{$\begin{array}{l}N=10^{3} \\
0.20077\end{array}$} & \multirow{2}{*}{$\begin{array}{l}N=10^{4} \\
0.22431\end{array}$} & \multirow{2}{*}{$\begin{array}{l}N=10^{5} \\
0.25085\end{array}$} \\
\hline & $1,431,993$ & $P_{\mathrm{f}}$ & & & & & & \\
\hline \multirow{2}{*}{$\begin{array}{c}\sigma_{\mathrm{S}}^{1}=245 \mathrm{MPa} \\
N=10\end{array}$} & $N_{\mathrm{f}}$ & & $N=1$ & $N=10$ & $N=10^{2}$ & $N=10^{3}$ & $N=10^{4}$ & $N=10^{5}$ \\
\hline & $1,225,895$ & $P_{\mathrm{f}}$ & 0.00609 & 0.02329 & 0.18207 & 0.20294 & 0.22648 & 0.25302 \\
\hline \multirow{2}{*}{$\begin{array}{c}\sigma_{\mathrm{s}}=245 \mathrm{MPa} \\
\quad N=10^{2}\end{array}$} & $N_{\mathrm{f}}$ & & $N=1$ & $N=10$ & $N=10^{2}$ & $N=10^{3}$ & $N=10^{4}$ & $N=10^{5}$ \\
\hline & 418,087 & $P_{\mathrm{f}}$ & 0.00609 & 0.02113 & 0.19662 & 0.21749 & 0.24103 & 0.26757 \\
\hline \multirow{2}{*}{$\begin{array}{c}\sigma_{\mathrm{s}}=245 \mathrm{MPa} \\
N=10^{3}\end{array}$} & $N_{\mathrm{f}}$ & & $N=1$ & $N=10$ & $N=10^{2}$ & $N=10^{3}$ & $N=10^{4}$ & $N=10^{5}$ \\
\hline & 368,186 & $P_{\mathrm{f}}$ & 0.00609 & 0.02113 & 0.17991 & 0.21915 & 0.24269 & 0.26923 \\
\hline \multirow{2}{*}{$\begin{array}{c}\sigma_{\mathrm{s}}=245 \mathrm{MPa} \\
N=10^{4}\end{array}$} & $N_{\mathrm{f}}$ & & $N=1$ & $N=10$ & $N=10^{2}$ & $N=10^{3}$ & $N=10^{4}$ & $N=10^{5}$ \\
\hline & 320,486 & $P_{\mathrm{f}}$ & 0.00609 & 0.02113 & 0.17991 & 0.20077 & 0.2445 & 0.27104 \\
\hline \multirow{2}{*}{$\begin{array}{c}\sigma_{\mathrm{s}}=245 \mathrm{MPa} \\
N=10^{5}\end{array}$} & $N_{\mathrm{f}}$ & & $N=1$ & $N=10$ & $N=10^{2}$ & $N=10^{3}$ & $N=10^{4}$ & $N=10^{5}$ \\
\hline & 275,798 & $P_{\mathrm{f}}$ & 0.00609 & 0.02113 & 0.17991 & 0.20077 & 0.22431 & 0.27298 \\
\hline \multirow{2}{*}{$\begin{array}{c}\sigma_{\mathrm{s}}=250 \mathrm{MPa} \\
N=10\end{array}$} & $N_{\mathrm{f}}$ & & $N=1$ & $N=10$ & $N=10^{2}$ & $N=10^{3}$ & $N=10^{4}$ & $N=10^{5}$ \\
\hline & 989,365 & $P_{\mathrm{f}}$ & 0.00609 & 0.02626 & 0.18504 & 0.2059 & 0.22944 & 0.25598 \\
\hline \multirow{2}{*}{$\begin{array}{c}\sigma_{\mathrm{s}}=250 \mathrm{MPa} \\
N=10^{2}\end{array}$} & $N_{\mathrm{f}}$ & & $N=1$ & $N=10$ & $N=10^{2}$ & $N=10^{3}$ & $N=10^{4}$ & $N=10^{5}$ \\
\hline & 70,700 & $P_{\mathrm{f}}$ & 0.00609 & 0.02113 & 0.21897 & 0.23983 & 0.26338 & 0.28571 \\
\hline \multirow{2}{*}{$\begin{array}{c}\sigma_{\mathrm{s}}=250 \mathrm{MPa} \\
N=10^{3}\end{array}$} & $N_{\mathrm{f}}$ & & $N=1$ & $N=10$ & $N=10^{2}$ & $N=10^{3}$ & $N=10^{4}$ & $N=10^{5}$ \\
\hline & 51,311 & $P_{\mathrm{f}}$ & 0.00609 & 0.02113 & 0.17991 & 0.24365 & 0.26719 & 0.28571 \\
\hline \multirow{2}{*}{$\begin{array}{c}\sigma_{\mathrm{s}}=250 \mathrm{MPa} \\
N=10^{4}\end{array}$} & $N_{\mathrm{f}}$ & & $N=1$ & $N=10$ & $N=10^{2}$ & $N=10^{3}$ & $N=10^{4}$ & $N=10^{5}$ \\
\hline & 36,095 & $P_{\mathrm{f}}$ & 0.00609 & 0.02113 & 0.17991 & 0.20077 & 0.27131 & 0.28571 \\
\hline \multirow{2}{*}{$\begin{array}{c}\sigma_{\mathrm{s}}=255 \mathrm{MPa} \\
N=10\end{array}$} & $N_{\mathrm{f}}$ & & $N=1$ & $N=10$ & $N=10^{2}$ & $N=10^{3}$ & $N=10^{4}$ & $N=10^{5}$ \\
\hline & 779,144 & $P_{\mathrm{f}}$ & 0.00609 & 0.02952 & 0.1883 & 0.20917 & 0.23271 & 0.25925 \\
\hline \multirow{2}{*}{$\begin{array}{c}\sigma_{\mathrm{s}}=255 \mathrm{MPa} \\
N=10^{2}\end{array}$} & $N_{\mathrm{f}}$ & & $N=1$ & $N=10$ & $N=10^{2}$ & $N=10^{3}$ & $N=8593$ & \\
\hline & 8593 & $P_{\mathrm{f}}$ & 0.00609 & 0.02113 & 0.24295 & 0.26381 & 0.28571 & \\
\hline \multirow{2}{*}{$\begin{array}{c}\sigma_{\mathrm{s}}=255 \mathrm{MPa} \\
N=10^{3}\end{array}$} & $N_{\mathrm{f}}$ & & $N=1$ & $N=10$ & $N=10^{2}$ & $N=10^{3}$ & $N=4870$ & \\
\hline & 4870 & $P_{\mathrm{f}}$ & 0.00609 & 0.02113 & 0.17991 & 0.26984 & 0.28571 & \\
\hline
\end{tabular}

\footnotetext{
${ }^{1} \sigma_{\mathrm{s}}$ is stochastic overloading stress; ${ }^{2} N_{\mathrm{f}}$ is the cycle number corresponding to fatigue fracture; ${ }^{3} \mathrm{~N}$ is applied cycle.
}

\subsection{Cross-Ply C/SiC Composite}

The cross-ply $\mathrm{T}-700^{\mathrm{TM}}$ carbon fiber-reinforced silicon carbide composite was fabricated using the hot-pressing (HP) method, which offered the ability to fabricate dense composites via a liquid phase sintering method at a low temperature. The fiber volume is $V_{\mathrm{f}}=0.4$, and the average tensile strength is approximately $\sigma_{\text {uts }}=124 \mathrm{MPa}$. The dog-bone shaped specimens were cut from $150 \mathrm{~mm} \times 150 \mathrm{~mm}$ panels by water cutting. The tension-tension fatigue tests were conducted on an MTS Model 809 servo hydraulic load-frame (MTS Systems Corp., Minneapolis, MN, USA). The fatigue experiments were in a sinusoidal wave form with a loading frequency $f=10 \mathrm{~Hz}$. The fatigue load ratio $\left(\sigma_{\min } / \sigma_{\max }\right)$ was $R=$ 0.1. The fatigue tests were conducted under load control at room temperature.

Figure 3 a shows experimental and predicted fatigue life S-N curves of the cross-ply C/SiC composite. When the fatigue limit applied cycle number is defined to be $N_{\text {limit }}=10^{6}$, the corresponding predicted fatigue limit stress is approximately $\sigma_{\text {limit }}=103 \mathrm{MPa}$ (approximately $83 \% \sigma_{\mathrm{uts}}$ ). 

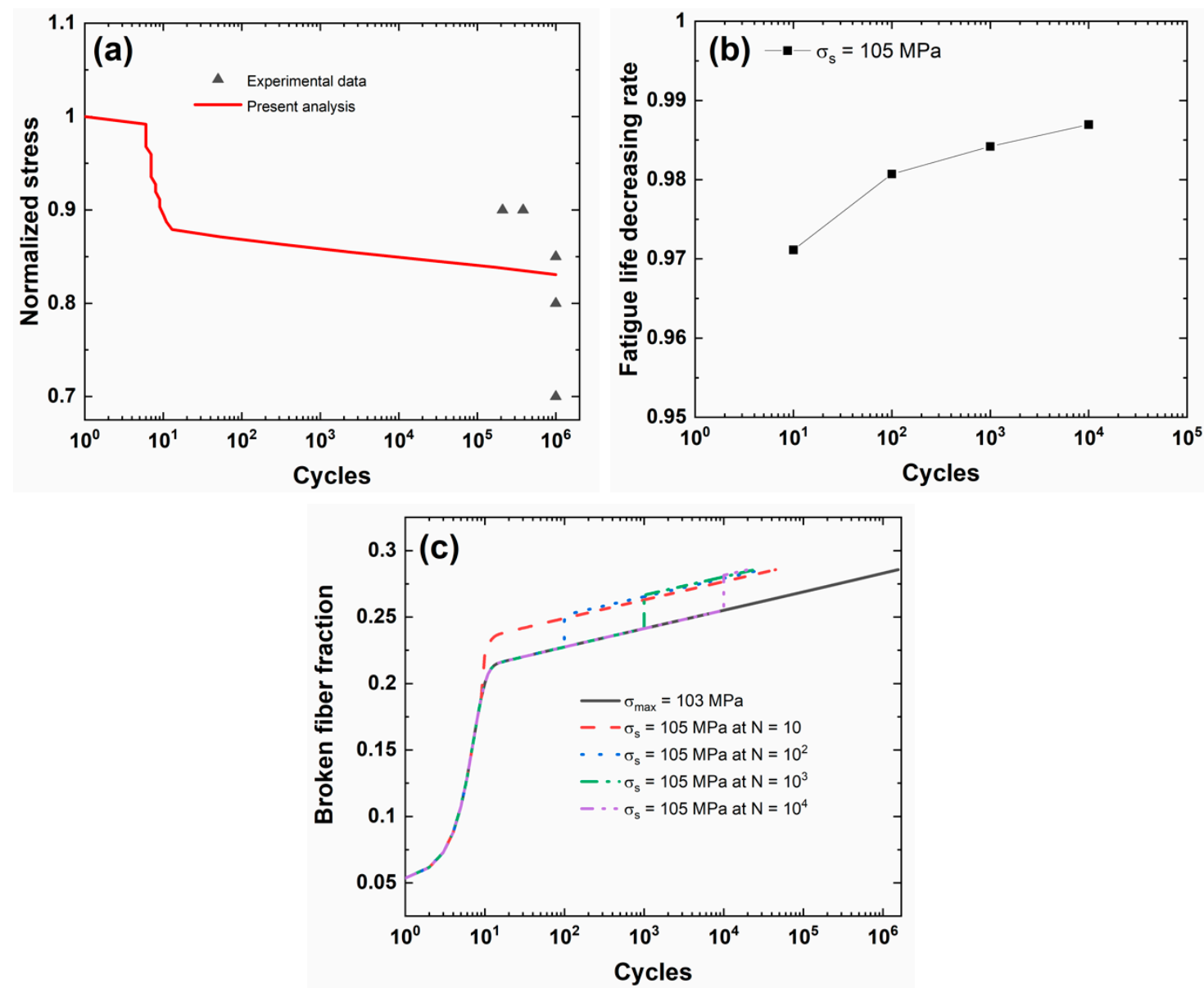

Figure 3. (a) Experimental and predicted fatigue life S-N curves; (b) the fatigue life decreasing rate versus occurrence applied cycle curve; and, (c) the broken fiber fraction versus applied cycle curves under $\sigma_{\text {limit }}=103 \mathrm{MPa}$ and stochastic overloading stress $\sigma_{\mathrm{s}}=105 \mathrm{MPa}$ at $N_{\mathrm{s}}=10,10^{2}, 10^{3}$, and $10^{4}$ of cross-ply $\mathrm{C} / \mathrm{SiC}$ composite.

Figure $3 \mathrm{~b}$ shows the fatigue life decreasing rate versus the occurrence applied cycle number of stochastic overloading stress curves under $\sigma_{\mathrm{s}}=105 \mathrm{MPa}$ (approximately 1.019 fatigue limit stress). During the application of CMC components, the overloading stress level is not high, and the low overloading stress level is chosen for analysis. The fatigue life decreasing rate increases with the occurrence applied cycle number of stochastic overloading. Under $\sigma_{\mathrm{s}}=105 \mathrm{MPa}$, the fatigue life decreasing rate increases from $\Lambda=0.97114$ at $N_{\mathrm{s}}=10$ to $\Lambda=0.98696$ at $N_{\mathrm{s}}=10^{4}$. When the occurrence applied cycle number is between $N_{\mathrm{s}}=10$ and $10^{2}$, the fatigue life decreasing rate increases rapidly; however, when the occurrence applied cycle is between $N_{\mathrm{s}}=10^{2}$ and $10^{4}$, the fatigue life decreasing rate increases slowly. The occurrence of stochastic overloading stress at the initial stage of cyclic fatigue loading deteriorates the fatigue damage evolution, i.e., decreasing matrix crack spacing in transverse and longitudinal plies, increasing interface debonding length, and broken fiber fraction; however, when matrix cracking and interface wear approach a steady-state, the effect of stochastic overloading on the fatigue damage or fatigue life decreasing rate decreases.

Figure $3 c$ shows the broken fiber fraction versus applied cycle number curves for different occurrence cycle numbers (i.e., $N_{\mathrm{s}}=10,10^{2}, 10^{3}$, and $10^{4}$ ). The broken fiber fraction increases when stochastic overloading stress occurs, and the difference of the broken fiber fraction between the original peak stress and stochastic overloading stress level increases with the applied cycle.

Table 3 shows the fatigue limit stress and broken fiber fraction under a stochastic overloading stress of $\sigma_{\mathrm{s}}=105 \mathrm{MPa}$ at different occurrence applied cycle numbers. When stochastic overloading 
stress $\sigma_{\mathrm{s}}=105 \mathrm{MPa}$ occurs at applied cycle numbers of $N_{\mathrm{s}}=10,10^{2}, 10^{3}$, and $10^{4}$, the broken fiber fraction increases from $P_{\mathrm{f}}=0.20047,0.22748,0.24133$, and 0.25508 under $\sigma_{\text {limit }}=103 \mathrm{MPa}$ to $P_{\mathrm{f}}=$ $0.22205,0.25148,0.26653$, and 0.28143 .

Table 3. Fatigue limit stress and broken fiber fraction of cross-ply C/SiC composite under stochastic overloading stress.

\begin{tabular}{|c|c|c|c|c|c|c|c|c|}
\hline \multirow{2}{*}{$\sigma_{\max }=103 \mathrm{MPa}$} & Nf & & $\mathbf{N}=1$ & $\mathbf{N}=10$ & $\mathrm{~N}=102$ & $N=103$ & $\mathrm{~N}=104$ & $N=105$ \\
\hline & $1,568,296$ & $P_{\mathrm{f}}$ & 0.05349 & 0.20047 & 0.22748 & 0.24133 & 0.25508 & 0.26892 \\
\hline \multirow{2}{*}{$\begin{array}{c}\sigma_{\mathrm{s}}=105 \mathrm{MPa} \\
N=10\end{array}$} & $N_{\mathrm{f}}$ & & $N=1$ & $N=10$ & $N=10^{2}$ & $N=10^{3}$ & $N=10^{4}$ & $N=45,256$ \\
\hline & 45,256 & $P_{\mathrm{f}}$ & 0.05349 & 0.22205 & 0.24906 & 0.26291 & 0.27666 & 0.28571 \\
\hline \multirow{2}{*}{$\begin{array}{c}\sigma_{\mathrm{s}}=105 \mathrm{MPa} \\
N=10^{2}\end{array}$} & $N_{\mathrm{f}}$ & & $N=1$ & $N=10$ & $N=10^{2}$ & $N=10^{3}$ & $N=10^{4}$ & $N=30,243$ \\
\hline & 30,243 & $P_{\mathrm{f}}$ & 0.05349 & 0.20047 & 0.25148 & 0.26533 & 0.27908 & 0.28571 \\
\hline \multirow{2}{*}{$\begin{array}{c}\sigma_{\mathrm{s}}=105 \mathrm{MPa} \\
N=10^{3}\end{array}$} & $N_{\mathrm{f}}$ & & $N=1$ & $N=10$ & $N=10^{2}$ & $N=10^{3}$ & $N=10^{4}$ & $N=24,787$ \\
\hline & 24,787 & $P_{\mathrm{f}}$ & 0.05349 & 0.20047 & 0.22748 & 0.26653 & 0.28028 & 0.28571 \\
\hline \multirow{2}{*}{$\begin{array}{c}\sigma_{\mathrm{s}}=105 \mathrm{MPa} \\
N=10^{4}\end{array}$} & $N_{\mathrm{f}}$ & & $N=1$ & $N=10$ & $N=10^{2}$ & $N=10^{3}$ & $N=10^{4}$ & $N=20,455$ \\
\hline & 20,455 & $P_{\mathrm{f}}$ & 0.05349 & 0.20047 & 0.22748 & 0.24133 & 0.28143 & 0.28571 \\
\hline
\end{tabular}

\subsection{The $2 \mathrm{DC} / \mathrm{SiC}$ Composite}

The $2 \mathrm{D} \mathrm{T}-300^{\mathrm{TM}}$ carbon fiber-reinforced silicon carbide composite was fabricated using the chemical vapor infiltration (CVI) method. It contained 26 plies of plain-weave cloth in a $\left(0^{\circ} / 90^{\circ}\right)$ lay-up. Fiber preform was given a pyrolytic carbon coating. The fiber volume was $45 \%$, and density was $1.93-1.98 \mathrm{~g} / \mathrm{cm}^{3}$, and the porosity was approximately $22 \%$. The dog-bone shaped specimens were cut from $200 \mathrm{~mm} \times 200 \mathrm{~mm}$ panels using diamond tooling. The tension-tension fatigue tests at room temperature were conducted on a servohydraulic load-frame that was equipped with edge-loaded grips. The fatigue experiments were performed under load control at a sinusoidal wave form and a loading frequency $f=10 \mathrm{~Hz}$. The fatigue load ratio $\left(\sigma_{\min } / \sigma_{\max }\right)$ was $R=0.1$. The average tensile strength was approximately $\sigma_{\text {uts }}=420 \mathrm{MPa}$.

Figure $4 \mathrm{a}$ shows experimental and predicted fatigue life $\mathrm{S}-\mathrm{N}$ curves of the $2 \mathrm{D} \mathrm{C} / \mathrm{SiC}$ composite. When the fatigue limit applied cycle number is defined to be $N_{\text {limit }}=10^{6}$, the corresponding predicted fatigue limit stress is approximately $\sigma_{\text {limit }}=348 \mathrm{MPa}$ (approximately $82.8 \% \sigma_{\mathrm{uts}}$ ).

Figure $4 \mathrm{~b}$ shows the fatigue life decreasing rate versus the occurrence applied cycle number of stochastic overloading stress curves for different stochastic overloading stress levels of $\sigma_{\mathrm{s}}=350,355$, and $360 \mathrm{MPa}$ (i.e., approximately 1.005, 1.02, 1.034 fatigue limit stress). During the application of CMC components, the overloading stress level is not high, and the low overloading stress level is chosen for analysis. Under the same stochastic overloading stress level, the fatigue life decreasing rate increases with the occurrence applied cycle of stochastic overloading. Under $\sigma_{\mathrm{s}}=350 \mathrm{MPa}$, the fatigue life decreasing rate increases from $\Lambda=0.03699$ at $N_{\mathrm{s}}=10$ to $\Lambda=0.21481$ at $N_{\mathrm{s}}=10^{5} ;$ under $\sigma_{\mathrm{s}}=355 \mathrm{MPa}$, the fatigue life decreasing rate increases from $\Lambda=0.12826$ at $N_{\mathrm{s}}=10$ to $\Lambda=0.59226$ at $N_{\mathrm{s}}=10^{5}$; under $\sigma_{\mathrm{s}}=360 \mathrm{MPa}$, the fatigue life decreasing rate increases from $\Lambda=0.21723$ at $N_{\mathrm{s}}=10$ to $\Lambda=0.80517$ at $N_{\mathrm{s}}=10^{5}$. When the occurrence applied cycle is between $N_{\mathrm{s}}=10$ and $10^{2}$, the fatigue life decreasing rate increases slowly with the occurrence applied cycle; however, when the occurrence applied cycle is between $N_{\mathrm{S}}=10^{2}$ and $10^{5}$, the fatigue life decreasing rate increases rapidly with the applied cycle. For $2 \mathrm{D} \mathrm{C} / \mathrm{SiC}$, the fatigue damage is not sensitive to stochastic overloading stress at the initial stage of cyclic fatigue loading; however, with the applied cycles increasing, the fatigue damage extent increases, leading to the rapid increase in the fatigue life decreasing rate with the occurrence cycle number of the stochastic overloading stress. 

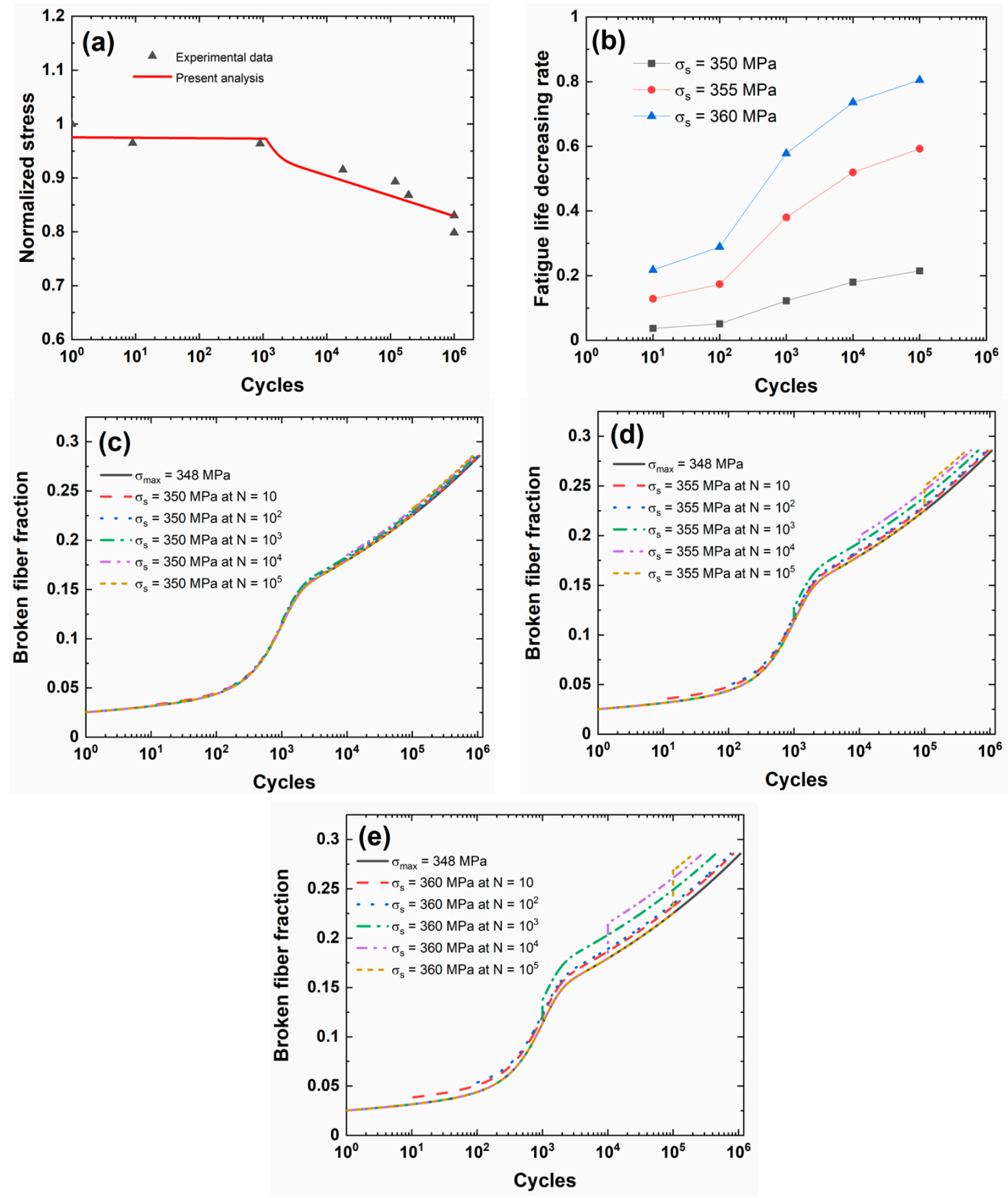

Figure 4. (a) Experimental and predicted fatigue life S-N curves; (b) the fatigue life decreasing rate versus occurrence applied cycle curve for different stochastic overloading stress levels; (c) the broken fiber fraction versus applied cycle curves under $\sigma_{\text {limit }}=348 \mathrm{MPa}$ and stochastic overloading stress $\sigma_{\mathrm{s}}=$ $350 \mathrm{MPa}$ at $N_{\mathrm{S}}=10,10^{2}, 10^{3}, 10^{4}$, and $10^{5} ;(\mathbf{d})$ the broken fiber fraction versus applied cycle curves under $\sigma_{\text {limit }}=348 \mathrm{MPa}$ and stochastic overloading stress $\sigma_{\mathrm{s}}=355 \mathrm{MPa}$ at $N_{\mathrm{s}}=10,10^{2}, 10^{3}, 10^{4}$, and $10^{5}$; and, (e) the broken fiber fraction versus applied cycle curves under $\sigma_{\text {limit }}=348 \mathrm{MPa}$ and stochastic overloading stress $\sigma_{\mathrm{s}}=360 \mathrm{MPa}$ at $N_{\mathrm{s}}=10,10^{2}, 10^{3}, 10^{4}$, and $10^{5}$ of $2 \mathrm{D} \mathrm{C} / \mathrm{SiC}$ composite.

Figure $4 \mathrm{c}-\mathrm{e}$ shows the broken fiber fraction versus the applied cycle number curves for different stochastic overloading stress levels and occurrence cycle numbers. The broken fiber fraction increases when stochastic overloading stress occurs, and the difference of the broken fiber fraction between the original peak stress and stochastic overloading stress level increases with the applied cycle. 
Table 4 shows the fatigue limit stress and broken fiber fraction at different occurrence cycle numbers and stochastic overloading stress. When stochastic overloading stress $\sigma_{\mathrm{s}}=350 \mathrm{MPa}$ occurs at applied cycles $N_{\mathrm{s}}=10,10^{2}, 10^{3}, 10^{4}$, and $10^{5}$, the broken fiber fraction increases from $P_{\mathrm{f}}=0.03153$, $0.04397,0.11358,0.1795$, and 0.22527 under $\sigma_{\text {limit }}=348 \mathrm{MPa}$ to $P_{\mathrm{f}}=0.03261,0.04547,0.11731,0.18516$, and 0.23215; when stochastic overloading stress $\sigma_{\mathrm{s}}=355 \mathrm{MPa}$ occurs at applied cycles $N_{\mathrm{s}}=10,10^{2}, 10^{3}$, $10^{4}$, and $10^{5}$, the broken fiber fraction increases to $P_{\mathrm{f}}=0.03546,0.04941,0.12704,0.19983$, and 0.24996; finally, when stochastic overloading stress $\sigma_{\mathrm{S}}=360 \mathrm{MPa}$ occurs at applied cycles $N=10,10^{2}, 10^{3}, 10^{4}$, and $10^{5}$, the broken fiber fraction increases to $P_{\mathrm{f}}=0.0385,0.05361,0.13736,0.2153$, and 0.26861.

Table 4. Fatigue limit stress and broken fiber fraction of $2 \mathrm{D} \mathrm{C} / \mathrm{SiC}$ composite under stochastic overloading stress.

\begin{tabular}{|c|c|c|c|c|c|c|c|c|}
\hline \multirow{2}{*}{$\sigma_{\max }=348 \mathrm{MPa}$} & \multicolumn{2}{|l|}{$N_{\mathrm{f}}$} & $N=1$ & \multirow{2}{*}{$\begin{array}{l}N=10 \\
0.03153\end{array}$} & \multirow{2}{*}{$\begin{array}{l}N=10^{2} \\
0.04397\end{array}$} & \multirow{2}{*}{$\begin{array}{l}N=10^{3} \\
0.11358\end{array}$} & \multirow{2}{*}{$\begin{array}{c}N=10^{4} \\
0.1795\end{array}$} & \multirow{2}{*}{$\begin{array}{l}N=10^{5} \\
0.22527\end{array}$} \\
\hline & $1,067,612$ & $P_{\mathrm{f}}$ & 0.02529 & & & & & \\
\hline \multirow{2}{*}{$\begin{array}{c}\sigma_{\mathrm{s}}=350 \mathrm{MPa} \\
N=10\end{array}$} & $N_{\mathrm{f}}$ & & $N=1$ & $N=10$ & $N=10^{2}$ & $N=10^{3}$ & $N=10^{4}$ & $N=10^{5}$ \\
\hline & $1,028,121$ & $P_{\mathrm{f}}$ & 0.02529 & 0.03261 & 0.04505 & 0.11466 & 0.18059 & 0.22635 \\
\hline \multirow{2}{*}{$\begin{array}{c}\sigma_{\mathrm{s}}=350 \mathrm{MPa} \\
N=10^{2}\end{array}$} & $N_{\mathrm{f}}$ & & $N=1$ & $N=10$ & $N=10^{2}$ & $N=10^{3}$ & $N=10^{4}$ & $N=10^{5}$ \\
\hline & $1,013,262$ & $P_{\mathrm{f}}$ & 0.02529 & 0.03153 & 0.04547 & 0.11508 & 0.18101 & 0.22677 \\
\hline \multirow{2}{*}{$\begin{array}{c}\sigma_{\mathrm{s}}=350 \mathrm{MPa} \\
N=10^{3}\end{array}$} & $N_{\mathrm{f}}$ & & $N=1$ & $N=10$ & $N=10^{2}$ & $N=10^{3}$ & $N=10^{4}$ & $N=10^{5}$ \\
\hline & 937,226 & $P_{\mathrm{f}}$ & 0.02529 & 0.03153 & 0.04397 & 0.11731 & 0.18323 & 0.229 \\
\hline \multirow{2}{*}{$\begin{array}{c}\sigma_{\mathrm{s}}=350 \mathrm{MPa} \\
N=10^{4}\end{array}$} & $N_{\mathrm{f}}$ & & $N=1$ & $N=10$ & $N=10^{2}$ & $N=10^{3}$ & $N=10^{4}$ & $N=10^{5}$ \\
\hline & 875,603 & $P_{\mathrm{f}}$ & 0.02529 & 0.03153 & 0.04397 & 0.11358 & 0.18516 & 0.23093 \\
\hline \multirow{2}{*}{$\begin{array}{c}\sigma_{\mathrm{s}}=350 \mathrm{MPa} \\
N=10^{5}\end{array}$} & $N_{\mathrm{f}}$ & & $N=1$ & $N=10$ & $N=10^{2}$ & $N=10^{3}$ & $N=10^{4}$ & $N=10^{5}$ \\
\hline & 838,274 & $P_{\mathrm{f}}$ & 0.02529 & 0.03153 & 0.04397 & 0.11358 & 0.1795 & 0.23215 \\
\hline \multirow{2}{*}{$\begin{array}{c}\sigma_{\mathrm{s}}=355 \mathrm{MPa} \\
N=10\end{array}$} & $N_{\mathrm{f}}$ & & $N=1$ & $N=10$ & $N=10^{2}$ & $N=10^{3}$ & $N=10^{4}$ & $N=10^{5}$ \\
\hline & 930,683 & $P_{\mathrm{f}}$ & 0.02529 & 0.03546 & 0.0479 & 0.11751 & 0.18342 & 0.2292 \\
\hline \multirow{2}{*}{$\begin{array}{c}\sigma_{\mathrm{s}}=355 \mathrm{MPa} \\
N=10^{2}\end{array}$} & $N_{\mathrm{f}}$ & & $N=1$ & $N=10$ & $N=10^{2}$ & $N=10^{3}$ & $N=10^{4}$ & $N=10^{5}$ \\
\hline & 882,390 & $P_{\mathrm{f}}$ & 0.02529 & 0.03153 & 0.04941 & 0.11902 & 0.18493 & 0.23071 \\
\hline \multirow{2}{*}{$\begin{array}{c}\sigma_{\mathrm{s}}=355 \mathrm{MPa} \\
N=10^{3}\end{array}$} & $N_{\mathrm{f}}$ & & $N=1$ & $N=10$ & $N=10^{2}$ & $N=10^{3}$ & $N=10^{4}$ & $N=10^{5}$ \\
\hline & 661,586 & $P_{\mathrm{f}}$ & 0.02529 & 0.03153 & 0.04397 & 0.12704 & 0.19295 & 0.23873 \\
\hline \multirow{2}{*}{$\begin{array}{c}\sigma_{\mathrm{s}}=355 \mathrm{MPa} \\
N=10^{4}\end{array}$} & $N_{\mathrm{f}}$ & & $N=1$ & $N=10$ & $N=10^{2}$ & $N=10^{3}$ & $N=10^{4}$ & $N=10^{5}$ \\
\hline & 513,025 & $P_{\mathrm{f}}$ & 0.02529 & 0.03153 & 0.04397 & 0.11358 & 0.19983 & 0.24561 \\
\hline \multirow{2}{*}{$\begin{array}{c}\sigma_{\mathrm{s}}=355 \mathrm{MPa} \\
N=10^{5}\end{array}$} & $N_{\mathrm{f}}$ & & $N=1$ & $N=10$ & $N=10^{2}$ & $N=10^{3}$ & $N=10^{4}$ & $N=10^{5}$ \\
\hline & 435,308 & $P_{\mathrm{f}}$ & 0.02529 & 0.03153 & 0.04397 & 0.11358 & 0.17949 & 0.24996 \\
\hline \multirow{2}{*}{$\begin{array}{c}\sigma_{\mathrm{s}}=360 \mathrm{MPa} \\
N=10\end{array}$} & $N_{\mathrm{f}}$ & & $N=1$ & $N=10$ & $N=10^{2}$ & $N=10^{3}$ & $N=10^{4}$ & $N=10^{5}$ \\
\hline & 835,692 & $P_{\mathrm{f}}$ & 0.02529 & 0.0385 & 0.05094 & 0.12055 & 0.18646 & 0.23224 \\
\hline \multirow{2}{*}{$\begin{array}{c}\sigma_{\mathrm{S}}=360 \mathrm{MPa} \\
N=10^{2}\end{array}$} & $N_{\mathrm{f}}$ & & $N=1$ & $N=10$ & $N=10^{2}$ & $N=10^{3}$ & $N=10^{4}$ & $N=10^{5}$ \\
\hline & 759,497 & $P_{\mathrm{f}}$ & 0.02529 & 0.03153 & 0.05361 & 0.12323 & 0.18914 & 0.23491 \\
\hline \multirow{2}{*}{$\begin{array}{c}\sigma_{\mathrm{s}}=360 \mathrm{MPa} \\
N=10^{3}\end{array}$} & $N_{\mathrm{f}}$ & & $N=1$ & $N=10$ & $N=10^{2}$ & $N=10^{3}$ & $N=10^{4}$ & $N=10^{5}$ \\
\hline & 450,695 & $P_{\mathrm{f}}$ & 0.02529 & 0.03153 & 0.04397 & 0.13736 & 0.20327 & 0.24905 \\
\hline \multirow{2}{*}{$\begin{array}{c}\sigma_{\mathrm{s}}=360 \mathrm{MPa} \\
N=10^{4}\end{array}$} & $N_{\mathrm{f}}$ & & $N=1$ & $N=10$ & $N=10^{2}$ & $N=10^{3}$ & $N=10^{4}$ & $N=10^{5}$ \\
\hline & 282,293 & $P_{\mathrm{f}}$ & 0.02529 & 0.03153 & 0.04397 & 0.11358 & 0.2153 & 0.26108 \\
\hline \multirow{2}{*}{$\begin{array}{c}\sigma_{\mathrm{s}}=360 \mathrm{MPa} \\
N=10^{5}\end{array}$} & $N_{\mathrm{f}}$ & & $N=1$ & $N=10$ & $N=10^{2}$ & $N=10^{3}$ & $N=10^{4}$ & $N=10^{5}$ \\
\hline & 208,007 & $P_{\mathrm{f}}$ & 0.02529 & 0.03153 & 0.04397 & 0.11358 & 0.17949 & 0.26861 \\
\hline
\end{tabular}




\subsection{The 2.5D C/SiC Composite}

The 2.5D T-300 ${ }^{\mathrm{TM}}$ carbon fiber-reinforced silicon carbide composite was fabricated using the chemical vapor infiltration (CVI) method. Low pressure CVI was employed to deposit a pyrolytic carbon layer and a silicon matrix. A thin pyrolytic carbon layer was deposited on the surface of the carbon fiber as the interfacial layer with $\mathrm{C}_{3} \mathrm{H}_{8}$ at $800{ }^{\circ} \mathrm{C}$. Methyltrichlorosilane ( $\mathrm{MTS}, \mathrm{CH}_{3} \mathrm{SiCl}_{3}$ ) was used as a gas source for the deposition of the $\mathrm{SiC}$ matrix. The conditions for deposition were $1000^{\circ} \mathrm{C}$. Argon was employed as a diluent gas to slow down the chemical reaction rate of deposition. The test specimens were machined from fabricated composites and further coated with $\mathrm{SiC}$ by isothermal CVI under the same conditions. The fiber volume was $V_{\mathrm{f}}=0.4$, and the average tensile strength was approximately $\sigma_{\text {uts }}=225 \mathrm{MPa}$. The dog-bone shaped specimens were cut from composite panels using diamond tooling. The tension-tension fatigue tests were conducted on an MTS Model 809 servo hydraulic load-frame (MTS Systems Corp., Minneapolis, MN, USA). The fatigue experiments were performed under load control at a loading frequency $f=10 \mathrm{~Hz}$. The fatigue load ratio $\left(\sigma_{\min } / \sigma_{\max }\right)$ was $R=0.1$.

Figure 5a shows the experimental and predicted fatigue life $\mathrm{S}-\mathrm{N}$ curves of the $2.5 \mathrm{D} \mathrm{C} / \mathrm{SiC}$ composite. When the fatigue limit applied cycle number is defined to be $N_{\text {limit }}=10^{6}$, the corresponding predicted fatigue limit stress is approximately $\sigma_{\text {limit }}=143 \mathrm{MPa}$ (approximately $63.5 \% \sigma_{\text {uts }}$ ). The fatigue limit stress of the 2.5D C/SiC composite is lower than the other CMCs, i.e., unidirectional, cross-ply, 2D, and 3D CMCs. The low fatigue limit stress of the $2.5 \mathrm{D} \mathrm{C} / \mathrm{SiC}$ composite is mainly due to yarns bending inside of composites.

Figure $5 \mathrm{~b}$ shows the fatigue life decreasing rate versus the occurrence applied cycle number of stochastic overloading curves for different stochastic overloading stress levels of $\sigma_{\mathrm{s}}=145,150$, and $155 \mathrm{MPa}$ (i.e., 1.014, 1.049, 1.084 fatigue limit stress). During the application of CMC components, the overloading stress level is not high, and the low overloading stress level is chosen for analysis. Under the same stochastic overloading stress level, the fatigue life decreasing rate increases with the occurrence applied cycle of stochastic overloading stress. Under $\sigma_{\mathrm{s}}=145 \mathrm{MPa}$, the fatigue life decreasing rate increases from $\Lambda=0.00723$ at $N_{\mathrm{S}}=10$ to $\Lambda=0.11768$ at $N_{\mathrm{S}}=10^{5}$; under $\sigma_{\mathrm{s}}=150 \mathrm{MPa}$, the fatigue life decreasing rate increases from $\Lambda=0.02742$ at $N_{\mathrm{s}}=10$ to $\Lambda=0.3939$ at $N_{\mathrm{s}}=10^{5}$; and, under $\sigma_{\mathrm{s}}=155 \mathrm{MPa}$, the fatigue life decreasing rate increases from $\Lambda=0.05087$ at $N_{\mathrm{s}}=10$ to $\Lambda=$ 0.63095 at $N_{\mathrm{s}}=10^{5}$. For the $2.5 \mathrm{D} \mathrm{C} / \mathrm{SiC}$, with fatigue cycles increasing, the fatigue damage extent increases, leading to the increase in the fatigue life decreasing rate with the occurrence cycle number of stochastic overloading stress.

Figure $5 c-e$ shows the broken fiber fraction versus applied cycle curves for different stochastic overloading stress levels and occurrence applied cycle numbers. The broken fiber fraction increases when stochastic overloading stress occurs, and the difference of the broken fiber fraction between the original peak stress and stochastic overloading stress level increases with the applied cycle.

Table 5 shows the fatigue limit stress and broken fiber fraction at different occurrence applied cycle numbers and stochastic overloading stress. When stochastic overloading stress $\sigma_{\mathrm{s}}=145 \mathrm{MPa}$ occurs at applied cycles $N_{\mathrm{s}}=10,10^{2}, 10^{3}, 10^{4}$, and $10^{5}$, the broken fiber fraction increases from $P_{\mathrm{f}}=$ $0.00767,0.01251,0.03658,0.07519$, and 0.14038 under $\sigma_{\text {limit }}=143 \mathrm{MPa}$ to $P_{\mathrm{f}}=0.00834,0.01359,0.03969$, 0.08145 , and 0.15161 ; when stochastic overloading stress $\sigma_{\mathrm{s}}=150 \mathrm{MPa}$ occurs at applied cycles $N_{\mathrm{s}}=10$, $10^{2}, 10^{3}, 10^{4}$, and $10^{5}$, the broken fiber fraction increases to $P_{\mathrm{f}}=0.01021,0.01663,0.04842,0.09888$, and 0.1825 ; and, when stochastic overloading stress $\sigma_{\mathrm{s}}=155 \mathrm{MPa}$ occurs at applied cycles $N=10,10^{2}, 10^{3}$, $10^{4}$, and $10^{5}$, the broken fiber fraction increases to $P_{\mathrm{f}}=0.01241,0.02021,0.05864,0.11905$, and 0.21754 . 

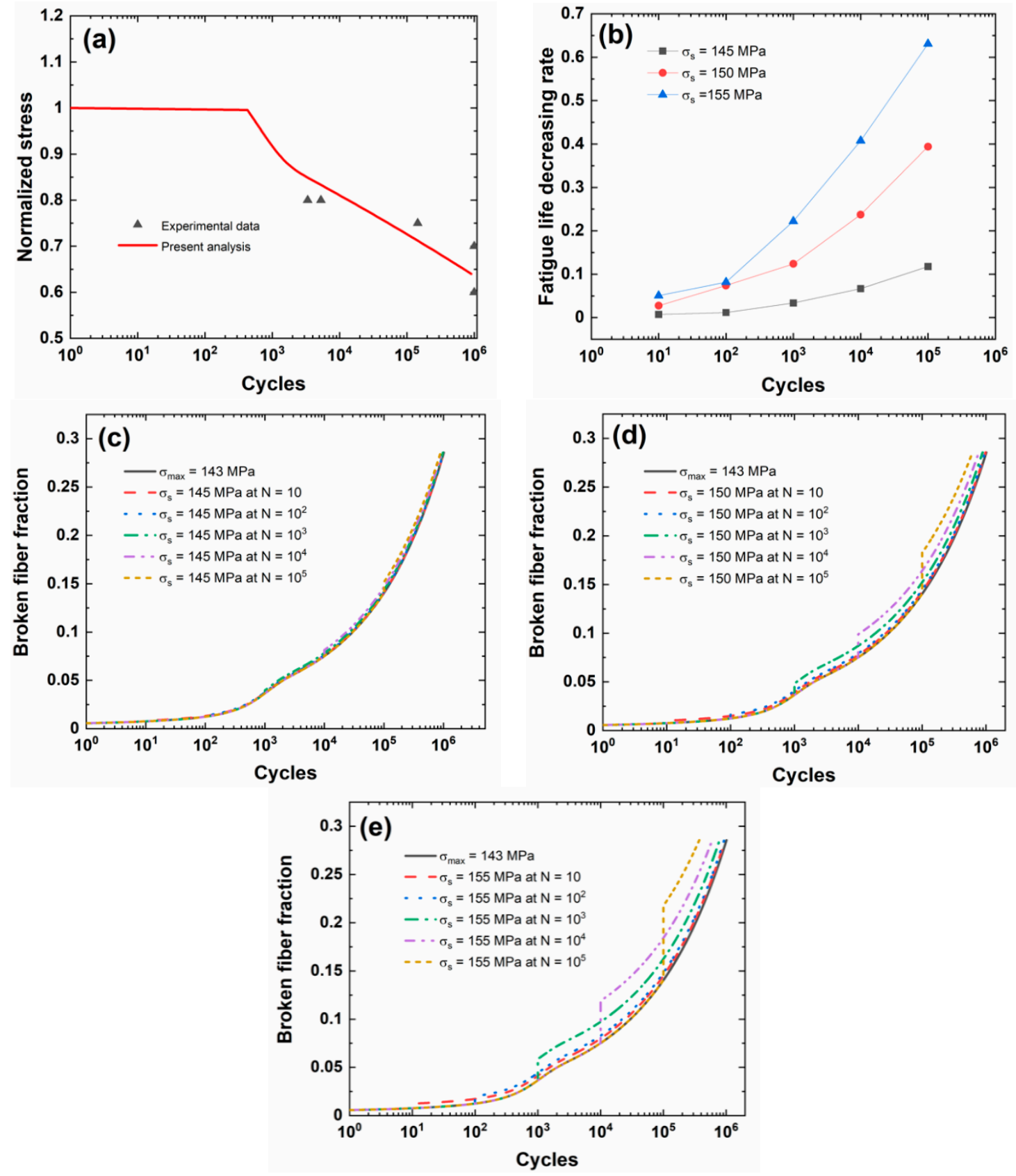

Figure 5. (a) Experimental and predicted fatigue life S-N curves; (b) the fatigue life decreasing rate versus occurrence applied cycle curve for different stochastic overloading stress levels; (c) the broken fiber fraction versus applied cycle curves under $\sigma_{\text {limit }}=143 \mathrm{MPa}$ and stochastic overloading stress of $\sigma_{\mathrm{S}}$ $=145 \mathrm{MPa}$ at $N_{\mathrm{s}}=10,10^{2}, 10^{3}, 10^{4}$, and $10^{5} ;(\mathbf{d})$ the broken fiber fraction versus applied cycle curves under $\sigma_{\text {limit }}=143 \mathrm{MPa}$ and stochastic overloading stress of $\sigma_{\mathrm{s}}=150 \mathrm{MPa}$ at $N_{\mathrm{s}}=10,10^{2}, 10^{3}, 10^{4}$, and $10^{5}$; and, (e) the broken fiber fraction versus applied cycle curves under $\sigma_{\text {limit }}=143 \mathrm{MPa}$ and stochastic overloading stress of $\sigma_{\mathrm{S}}=155 \mathrm{MPa}$ at $N_{\mathrm{S}}=10,10^{2}, 10^{3}, 10^{4}$, and $10^{5}$ of $2.5 \mathrm{D} \mathrm{C} / \mathrm{SiC}$ composite. 
Table 5. Fatigue limit stress and broken fiber fraction of 2.5D C/SiC composite under stochastic overloading stress.

\begin{tabular}{|c|c|c|c|c|c|c|c|c|}
\hline \multirow{2}{*}{$\sigma_{\max }=143 \mathrm{MPa}$} & \multicolumn{2}{|l|}{$N_{\mathrm{f}}$} & $N=1$ & \multirow{2}{*}{$\begin{array}{l}N=10 \\
0.00767\end{array}$} & \multirow{2}{*}{$\begin{array}{l}N=10^{2} \\
0.01251\end{array}$} & \multirow{2}{*}{$\begin{array}{l}N=10^{3} \\
0.03658\end{array}$} & \multirow{2}{*}{$\begin{array}{l}N=10^{4} \\
0.07519\end{array}$} & \multirow{2}{*}{$\begin{array}{l}N=10^{5} \\
0.14038\end{array}$} \\
\hline & $1,012,346$ & $P_{\mathrm{f}}$ & 0.00574 & & & & & \\
\hline \multirow{2}{*}{$\begin{array}{c}\sigma_{\mathrm{s}}=145 \mathrm{MPa} \\
N=10\end{array}$} & $N_{\mathrm{f}}$ & & $N=1$ & $N=10$ & $N=10^{2}$ & $N=10^{3}$ & $N=10^{4}$ & $N=10^{5}$ \\
\hline & $1,005,026$ & $P_{\mathrm{f}}$ & 0.00574 & 0.00834 & 0.01317 & 0.03724 & 0.07585 & 0.14105 \\
\hline \multirow{2}{*}{$\begin{array}{c}\sigma_{\mathrm{s}}=145 \mathrm{MPa} \\
N=10^{2}\end{array}$} & $N_{\mathrm{f}}$ & & $N=1$ & $N=10$ & $N=10^{2}$ & $N=10^{3}$ & $N=10^{4}$ & $N=10^{5}$ \\
\hline & $1,000,463$ & $P_{\mathrm{f}}$ & 0.00574 & 0.00767 & 0.01359 & 0.03766 & 0.07627 & 0.14146 \\
\hline \multirow{2}{*}{$\begin{array}{c}\sigma_{\mathrm{s}}=145 \mathrm{MPa} \\
N=10^{3}\end{array}$} & $N_{\mathrm{f}}$ & & $N=1$ & $N=10$ & $N=10^{2}$ & $N=10^{3}$ & $N=10^{4}$ & $N=10^{5}$ \\
\hline & 978,311 & $P_{\mathrm{f}}$ & 0.00574 & 0.00767 & 0.01251 & 0.03969 & 0.0783 & 0.1435 \\
\hline \multirow{2}{*}{$\begin{array}{c}\sigma_{\mathrm{S}}=145 \mathrm{MPa} \\
N=10^{4}\end{array}$} & $N_{\mathrm{f}}$ & & $N=1$ & $N=10$ & $N=10^{2}$ & $N=10^{3}$ & $N=10^{4}$ & $N=10^{5}$ \\
\hline & 944,705 & $P_{\mathrm{f}}$ & 0.00574 & 0.00767 & 0.01251 & 0.03658 & 0.08145 & 0.14664 \\
\hline \multirow{2}{*}{$\begin{array}{c}\sigma_{\mathrm{s}}=145 \mathrm{MPa} \\
N=10^{5}\end{array}$} & $N_{\mathrm{f}}$ & & $N=1$ & $N=10$ & $N=10^{2}$ & $N=10^{3}$ & $N=10^{4}$ & $N=10^{5}$ \\
\hline & 893,212 & $P_{\mathrm{f}}$ & 0.00574 & 0.00767 & 0.01251 & 0.03658 & 0.07519 & 0.15161 \\
\hline \multirow{2}{*}{$\begin{array}{c}\sigma_{\mathrm{s}}=150 \mathrm{MPa} \\
N=10\end{array}$} & $N_{\mathrm{f}}$ & & $N=1$ & $N=10$ & $N=10^{2}$ & $N=10^{3}$ & $N=10^{4}$ & $N=10^{5}$ \\
\hline & 984,589 & $P_{\mathrm{f}}$ & 0.00574 & 0.01021 & 0.01504 & 0.03911 & 0.07772 & 0.14292 \\
\hline \multirow{2}{*}{$\begin{array}{c}\sigma_{\mathrm{s}}=150 \mathrm{MPa} \\
N=10^{2}\end{array}$} & $N_{\mathrm{f}}$ & & $N=1$ & $N=10$ & $N=10^{2}$ & $N=10^{3}$ & $N=10^{4}$ & $N=10^{5}$ \\
\hline & 967,500 & $P_{\mathrm{f}}$ & 0.00574 & 0.00767 & 0.01663 & 0.0407 & 0.07931 & 0.1445 \\
\hline \multirow{2}{*}{$\begin{array}{c}\sigma_{\mathrm{s}}=150 \mathrm{MPa} \\
N=10^{3}\end{array}$} & $N_{\mathrm{f}}$ & & $N=1$ & $N=10$ & $N=10^{2}$ & $N=10^{3}$ & $N=10^{4}$ & $N=10^{5}$ \\
\hline & 886,901 & $P_{\mathrm{f}}$ & 0.00574 & 0.00767 & 0.01251 & 0.04842 & 0.08703 & 0.15223 \\
\hline \multirow{2}{*}{$\begin{array}{c}\sigma_{\mathrm{s}}=150 \mathrm{MPa} \\
\quad N=10^{4}\end{array}$} & $N_{\mathrm{f}}$ & & $N=1$ & $N=10$ & $N=10^{2}$ & $N=10^{3}$ & $N=10^{4}$ & $N=10^{5}$ \\
\hline & 772,084 & $P_{\mathrm{f}}$ & 0.00574 & 0.00767 & 0.01251 & 0.03658 & 0.09888 & 0.16408 \\
\hline \multirow{2}{*}{$\begin{array}{c}\sigma_{\mathrm{s}}=150 \mathrm{MPa} \\
\quad N=10^{5}\end{array}$} & $N_{\mathrm{f}}$ & & $N=1$ & $N=10$ & $N=10^{2}$ & $N=10^{3}$ & $N=10^{4}$ & $N=10^{5}$ \\
\hline & 613,579 & $P_{\mathrm{f}}$ & 0.00574 & 0.00767 & 0.01251 & 0.03658 & 0.07519 & 0.1825 \\
\hline \multirow{2}{*}{$\begin{array}{c}\sigma_{\mathrm{s}}=155 \mathrm{MPa} \\
N=10\end{array}$} & $N_{\mathrm{f}}$ & & $N=1$ & $N=10$ & $N=10^{2}$ & $N=10^{3}$ & $N=10^{4}$ & $N=10^{5}$ \\
\hline & 960,848 & $P_{\mathrm{f}}$ & 0.00574 & 0.01241 & 0.01725 & 0.04132 & 0.07993 & 0.14512 \\
\hline \multirow{2}{*}{$\begin{array}{c}\sigma_{\mathrm{s}}=155 \mathrm{MPa} \\
N=10^{2}\end{array}$} & $N_{\mathrm{f}}$ & & $N=1$ & $N=10$ & $N=10^{2}$ & $N=10^{3}$ & $N=10^{4}$ & $N=10^{5}$ \\
\hline & 929,613 & $P_{\mathrm{f}}$ & 0.00574 & 0.00767 & 0.02021 & 0.04427 & 0.08288 & 0.14808 \\
\hline \multirow{2}{*}{$\begin{array}{c}\sigma_{\mathrm{s}}=155 \mathrm{MPa} \\
N=10^{3}\end{array}$} & $N_{\mathrm{f}}$ & & $N=1$ & $N=10$ & $N=10^{2}$ & $N=10^{3}$ & $N=10^{4}$ & $N=10^{5}$ \\
\hline & 787,301 & $P_{\mathrm{f}}$ & 0.00574 & 0.00767 & 0.01251 & 0.05864 & 0.09725 & 0.16245 \\
\hline \multirow{2}{*}{$\begin{array}{c}\sigma_{\mathrm{s}}=155 \mathrm{MPa} \\
N=10^{4}\end{array}$} & $N_{\mathrm{f}}$ & & $N=1$ & $N=10$ & $N=10^{2}$ & $N=10^{3}$ & $N=10^{4}$ & $N=10^{5}$ \\
\hline & 599,723 & $P_{\mathrm{f}}$ & 0.00574 & 0.00767 & 0.01251 & 0.03658 & 0.11905 & 0.18425 \\
\hline \multirow{2}{*}{$\begin{array}{c}\sigma_{\mathrm{s}}=155 \mathrm{MPa} \\
N=10^{5}\end{array}$} & $N_{\mathrm{f}}$ & & $N=1$ & $N=10$ & $N=10^{2}$ & $N=10^{3}$ & $N=10^{4}$ & $N=10^{5}$ \\
\hline & 373,608 & $P_{\mathrm{f}}$ & 0.00574 & 0.00767 & 0.01251 & 0.03658 & 0.07519 & 0.21754 \\
\hline
\end{tabular}

\subsection{The 3D C/SiC Composite}

The 3D T $-300^{\mathrm{TM}}$ carbon fiber-reinforced silicon carbide composite was fabricated using the chemical vapor infiltration (CVI) method. Low pressure I-CVI was employed to deposit a pyrolytic carbon layer and the silicon carbide matrix. A thin pyrolytic carbon layer was deposited on the surface of the carbon fiber as the interfacial layer with $\mathrm{C}_{4} \mathrm{H}_{10}$ at $950-1000{ }^{\circ} \mathrm{C}$. The thickness of the pyrolytic carbon layer was approximately $0.2 \mu \mathrm{m}$. The fiber volume is $V_{\mathrm{f}}=0.4$, and the average tensile strength is approximately $\sigma_{\text {uts }}=276 \mathrm{MPa}$. The dog-bone shaped specimens were cut from composite panels using the diamond tooling, and then coated with a $\mathrm{SiC}$ coating. The tension-tension fatigue tests at room temperature were conducted on a servohydraulic mechanical testing machine. The fatigue 
experiments were performed under load control at a loading frequency $f=60 \mathrm{~Hz}$. The fatigue load ratio $\left(\sigma_{\min } / \sigma_{\max }\right)$ was $R=0.1$. The loading frequency affects the fatigue life and fatigue limit stress. At room temperature, When the loading frequency increases, the fatigue limit stress also increases.

Figure 6 a shows experimental and predicted fatigue life $\mathrm{S}-\mathrm{N}$ curves of $3 \mathrm{D} \mathrm{C} / \mathrm{SiC}$ composite. When the fatigue limit applied cycle number is defined to be $N_{\text {limit }}=10^{6}$, the corresponding predicted fatigue limit stress is approximately $\sigma_{\text {limit }}=236 \mathrm{MPa}$ (approximately $85.5 \% \sigma_{\mathrm{uts}}$ ).
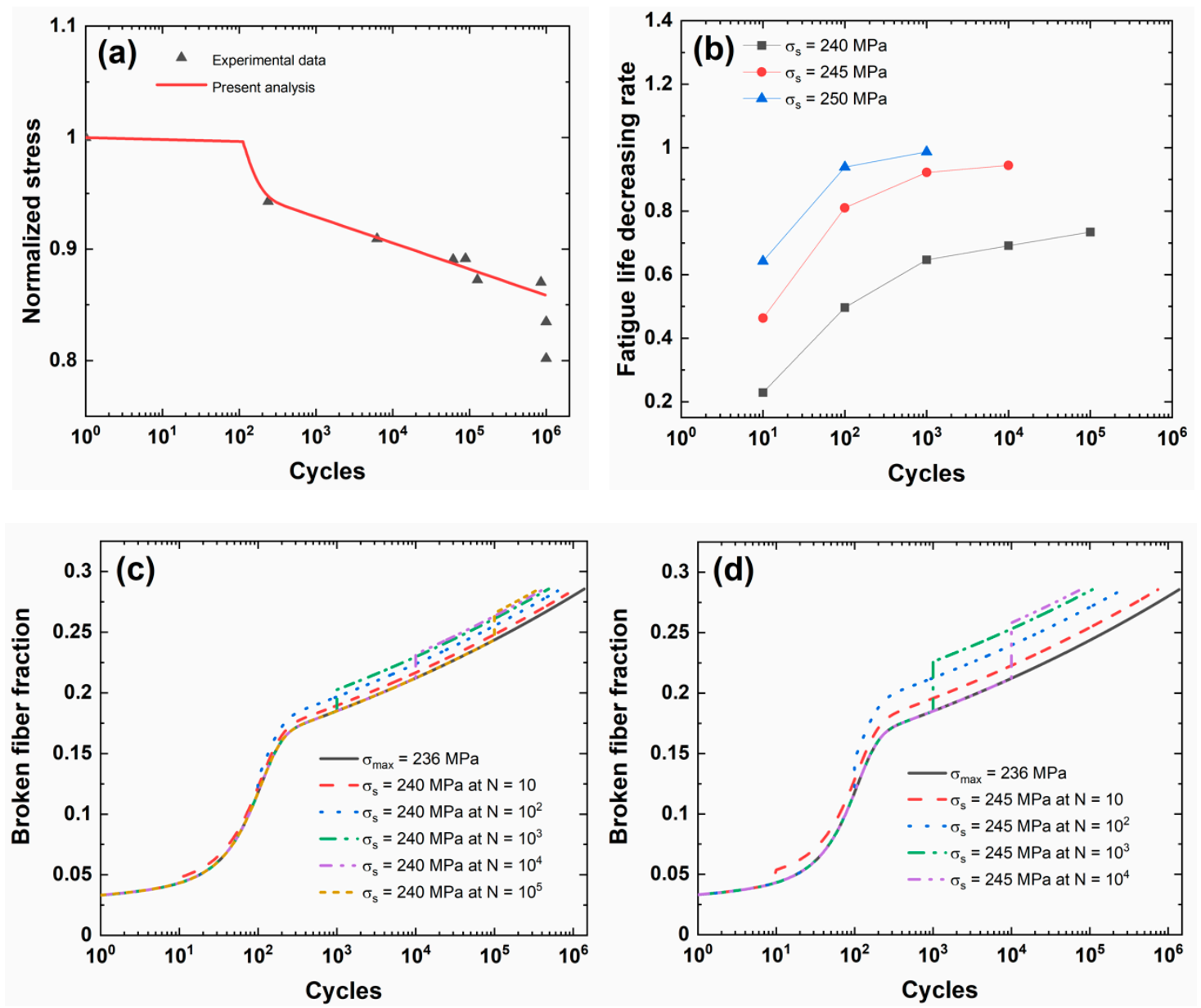

Figure 6. Cont. 


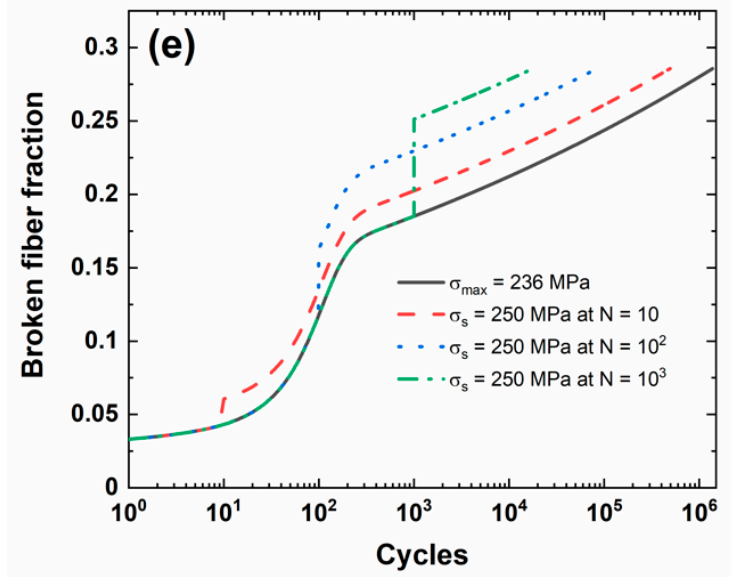

Figure 6. (a) Experimental and predicted fatigue life S-N curves; (b) the fatigue life decreasing rate versus occurrence applied cycle curve for different stochastic overloading stress levels; (c) the broken fiber fraction versus applied cycle curves under $\sigma_{\text {limit }}=236 \mathrm{MPa}$ and stochastic overloading stress of $\sigma_{\mathrm{S}}$ $=240 \mathrm{MPa}$ at $N_{\mathrm{s}}=10,10^{2}, 10^{3}, 10^{4}$, and $10^{5} ;(\mathbf{d})$ the broken fiber fraction versus applied cycle curves under $\sigma_{\text {limit }}=236 \mathrm{MPa}$ and stochastic overloading stress of $\sigma_{\mathrm{s}}=245 \mathrm{MPa}$ at $N_{\mathrm{s}}=10,10^{2}, 10^{3}$, and $10^{4}$; and, (e) the broken fiber fraction versus applied cycle curves under $\sigma_{\text {limit }}=236 \mathrm{MPa}$ and stochastic overloading stress of $\sigma_{\mathrm{s}}=250 \mathrm{MPa}$ at $N_{\mathrm{s}}=10,10^{2}$, and $10^{3}$ of 3D C/SiC composite.

Figure $6 \mathrm{~b}$ shows the fatigue life decreasing rate versus the occurrence cycle number of stochastic overloading curves for different stochastic overloading stress levels of $\sigma_{\mathrm{s}}=240,245$, and $250 \mathrm{MPa}$ (i.e., approximately 1.017, 1.038, and 1.059 fatigue limit stress). During application of CMC components, the overloading stress level is not high, and the low overloading stress level is chosen for analysis. Under the same stochastic overloading stress level, the fatigue life decreasing rate increases with the occurrence applied cycle of stochastic overloading. Under $\sigma_{\mathrm{s}}=240 \mathrm{MPa}$, the fatigue life decreasing rate increases from $\Lambda=0.22884$ at $N_{\mathrm{S}}=10$ to $\Lambda=0.73423$ at $N_{\mathrm{S}}=10^{5}$; under $\sigma_{\mathrm{s}}=245 \mathrm{MPa}$, the fatigue life decreasing rate increases from $\Lambda=0.46292$ at $N_{\mathrm{s}}=10$ to $\Lambda=0.94456$ at $N_{\mathrm{s}}=10^{4}$; and under $\sigma_{\mathrm{s}}$ $=250 \mathrm{MPa}$, the fatigue life decreasing rate increases from $\Lambda=0.64272$ at $N_{\mathrm{s}}=10$ to $\Lambda=0.98713$ at $N_{\mathrm{s}}=10^{3}$. When the occurrence applied cycle is between $N_{\mathrm{s}}=10$ and $10^{2}$, the fatigue life decreasing rate increases rapidly; however, when the occurrence applied cycle is between $N_{\mathrm{s}}=10^{2}$ and $10^{5}$, the fatigue life decreasing rate increases slowly. The occurrence of stochastic overloading stress at the initial stage of cyclic fatigue loading deteriorates the fatigue damage evolution, i.e., decreasing matrix crack spacing in transverse and longitudinal yarns, increasing interface debonding length, and broken fiber fraction; however, when matrix cracking and interface wear approach a steady-state, the effect of stochastic overloading on the fatigue damage or the fatigue life decreasing rate decreases.

Figure $6 c-e$ shows the broken fiber fraction versus the applied cycle curves for different stochastic overloading stress levels and occurrence cycle numbers. The broken fiber fraction increases when stochastic overloading stress occurs, and the difference of the broken fiber fraction between the original peak stress and stochastic overloading stress level increases with the applied cycle.

Table 6 shows the fatigue limit stress and broken fiber fraction at different occurrence cycle numbers and stochastic overloading stress. When stochastic overloading stress $\sigma_{\mathrm{S}}=240 \mathrm{MPa}$ occurs at applied cycles $N_{\mathrm{s}}=10,10^{2}, 10^{3}, 10^{4}$, and $10^{5}$, the broken fiber fraction increases from $P_{\mathrm{f}}=0.04324$, $0.11794,0.18509,0.2122$, and 0.24367 under $\sigma_{\text {limit }}=236 \mathrm{MPa}$ to $P_{\mathrm{f}}=0.04771,0.12961,0.2026,0.23189$, and 0.26575; when stochastic overloading stress $\sigma_{\mathrm{s}}=245 \mathrm{MPa}$ occurs at applied cycles $N_{\mathrm{s}}=10,10^{2}, 10^{3}$, and $10^{4}$, the broken fiber fraction increases to $P_{\mathrm{f}}=0.05383,0.14538,0.22602$, and 0.25812 ; and, when stochastic overloading stress $\sigma_{\mathrm{s}}=250 \mathrm{MPa}$ occurs at applied cycles $N=10,10^{2}$, and $10^{3}$, the broken fiber fraction increases to $P_{\mathrm{f}}=0.06055,0.1625$, and 0.25116 . 
Table 6. Fatigue limit stress and broken fiber fraction of 3D C/SiC composite under stochastic overloading stress.

\begin{tabular}{|c|c|c|c|c|c|c|c|c|}
\hline \multirow{2}{*}{$\sigma_{\max }=236 \mathrm{MPa}$} & $N_{\mathrm{f}}$ & & $N=1$ & $N=10$ & $N=10^{2}$ & $N=10^{3}$ & $N=10^{4}$ & $N=10^{5}$ \\
\hline & $1,383,192$ & $P_{\mathrm{f}}$ & 0.03314 & 0.04324 & 0.11794 & 0.18509 & 0.2122 & 0.24367 \\
\hline \multirow{2}{*}{$\begin{array}{c}\sigma_{\mathrm{s}}=240 \mathrm{MPa} \\
N=10\end{array}$} & $N_{\mathrm{f}}$ & & $N=1$ & $N=10$ & $N=10^{2}$ & $N=10^{3}$ & $N=10^{4}$ & $N=10^{5}$ \\
\hline & $1,066,666$ & $P_{\mathrm{f}}$ & 0.03314 & 0.04771 & 0.12242 & 0.18957 & 0.21668 & 0.24815 \\
\hline \multirow{2}{*}{$\begin{array}{c}\sigma_{\mathrm{s}}=240 \mathrm{MPa} \\
N=10^{2}\end{array}$} & $N_{\mathrm{f}}$ & & $N=1$ & $N=10$ & $N=10^{2}$ & $N=10^{3}$ & $N=10^{4}$ & $N=10^{5}$ \\
\hline & 696,321 & $P_{\mathrm{f}}$ & 0.03314 & 0.04324 & 0.12961 & 0.19676 & 0.22387 & 0.25534 \\
\hline \multirow{2}{*}{$\begin{array}{c}\sigma_{\mathrm{s}}=240 \mathrm{MPa} \\
N=10^{3}\end{array}$} & $N_{\mathrm{f}}$ & & $N=1$ & $N=10$ & $N=10^{2}$ & $N=10^{3}$ & $N=10^{4}$ & $N=10^{5}$ \\
\hline & 488,262 & $P_{\mathrm{f}}$ & 0.03314 & 0.04324 & 0.11794 & 0.2026 & 0.22971 & 0.26118 \\
\hline \multirow{2}{*}{$\begin{array}{c}\sigma_{\mathrm{s}}=240 \mathrm{MPa} \\
\quad N=10^{4}\end{array}$} & $N_{\mathrm{f}}$ & & $N=1$ & $N=10$ & $N=10^{2}$ & $N=10^{3}$ & $N=10^{4}$ & $N=10^{5}$ \\
\hline & 426,789 & $P_{\mathrm{f}}$ & 0.03314 & 0.04324 & 0.11794 & 0.18509 & 0.23189 & 0.26336 \\
\hline \multirow{2}{*}{$\begin{array}{c}\sigma_{\mathrm{s}}=240 \mathrm{MPa} \\
N=10^{5}\end{array}$} & $N_{\mathrm{f}}$ & & $N=1$ & $N=10$ & $N=10^{2}$ & $N=10^{3}$ & $N=10^{4}$ & $N=10^{5}$ \\
\hline & 367,609 & $P_{\mathrm{f}}$ & 0.03314 & 0.04324 & 0.11794 & 0.18509 & 0.2122 & 0.26575 \\
\hline \multirow{2}{*}{$\begin{array}{c}\sigma_{\mathrm{s}}=245 \mathrm{MPa} \\
N=10\end{array}$} & $N_{\mathrm{f}}$ & & $N=1$ & $N=10$ & $N=10^{2}$ & $N=10^{3}$ & $N=10^{4}$ & $N=10^{5}$ \\
\hline & 742,891 & $P_{\mathrm{f}}$ & 0.03314 & 0.05383 & 0.12853 & 0.19568 & 0.22279 & 0.25426 \\
\hline \multirow{2}{*}{$\begin{array}{c}\sigma_{\mathrm{s}}=245 \mathrm{MPa} \\
N=10^{2}\end{array}$} & $N_{\mathrm{f}}$ & & $N=1$ & $N=10$ & $N=10^{2}$ & $N=10^{3}$ & $N=10^{4}$ & $N=10^{5}$ \\
\hline & 262,035 & $P_{\mathrm{f}}$ & 0.03314 & 0.04324 & 0.14538 & 0.21253 & 0.23963 & 0.2711 \\
\hline \multirow{2}{*}{$\begin{array}{c}\sigma_{\mathrm{s}}=245 \mathrm{MPa} \\
N=10^{3}\end{array}$} & $N_{\mathrm{f}}$ & & $N=1$ & $N=10$ & $N=10^{2}$ & $N=10^{3}$ & $N=10^{4}$ & $N=10^{5}$ \\
\hline & 107,854 & $P_{\mathrm{f}}$ & 0.03314 & 0.04324 & 0.11794 & 0.22602 & 0.25313 & 0.2846 \\
\hline \multirow{2}{*}{$\begin{array}{c}\sigma_{\mathrm{S}}=245 \mathrm{MPa} \\
N=10^{4}\end{array}$} & $N_{\mathrm{f}}$ & & $N=1$ & $N=10$ & $N=10^{2}$ & $N=10^{3}$ & $N=10^{4}$ & $N=10^{5}$ \\
\hline & 76,689 & $P_{\mathrm{f}}$ & 0.03314 & 0.04324 & 0.11794 & 0.18509 & 0.25812 & 0.28571 \\
\hline \multirow{2}{*}{$\begin{array}{c}\sigma_{\mathrm{s}}=250 \mathrm{MPa} \\
N=10\end{array}$} & $N_{\mathrm{f}}$ & & $N=1$ & $N=10$ & $N=10^{2}$ & $N=10^{3}$ & $N=10^{4}$ & $N=10^{5}$ \\
\hline & 494,186 & $P_{\mathrm{f}}$ & 0.03314 & 0.06055 & 0.13525 & 0.2024 & 0.22951 & 0.26098 \\
\hline \multirow{2}{*}{$\begin{array}{c}\sigma_{\mathrm{s}}=250 \mathrm{MPa} \\
N=10^{2}\end{array}$} & $N_{\mathrm{f}}$ & & $N=1$ & $N=10$ & $N=10^{2}$ & $N=10^{3}$ & $N=10^{4}$ & $N=10^{5}$ \\
\hline & 84,191 & $P_{\mathrm{f}}$ & 0.03314 & 0.04324 & 0.1625 & 0.22966 & 0.25676 & 0.28571 \\
\hline \multirow{2}{*}{$\begin{array}{c}\sigma_{\mathrm{s}}=250 \mathrm{MPa} \\
N=10^{3}\end{array}$} & $N_{\mathrm{f}}$ & & $N=1$ & $N=10$ & $N=10^{2}$ & $N=10^{3}$ & $N=10^{4}$ & $N=10^{5}$ \\
\hline & 17,798 & $P_{\mathrm{f}}$ & 0.03314 & 0.04324 & 0.11794 & 0.25116 & 0.27827 & 0.28571 \\
\hline
\end{tabular}

\section{Discussion}

Figure 7 shows the fatigue life decreasing rate versus stochastic overloading stress for different occurrence cycle numbers of different $\mathrm{C} / \mathrm{SiC}$ composites. The fatigue life decreasing rate increases with the stochastic overloading stress level for different fiber preforms (i.e., unidirectional, cross-ply, 2D, $2.5 \mathrm{D}$, and 3D). However, with increasing applied cycles, the evolution of the fatigue life decreasing rate with stochastic overloading stress depends on the fiber preforms, which indicates that the fiber preforms affect the fatigue damage evolution process. 

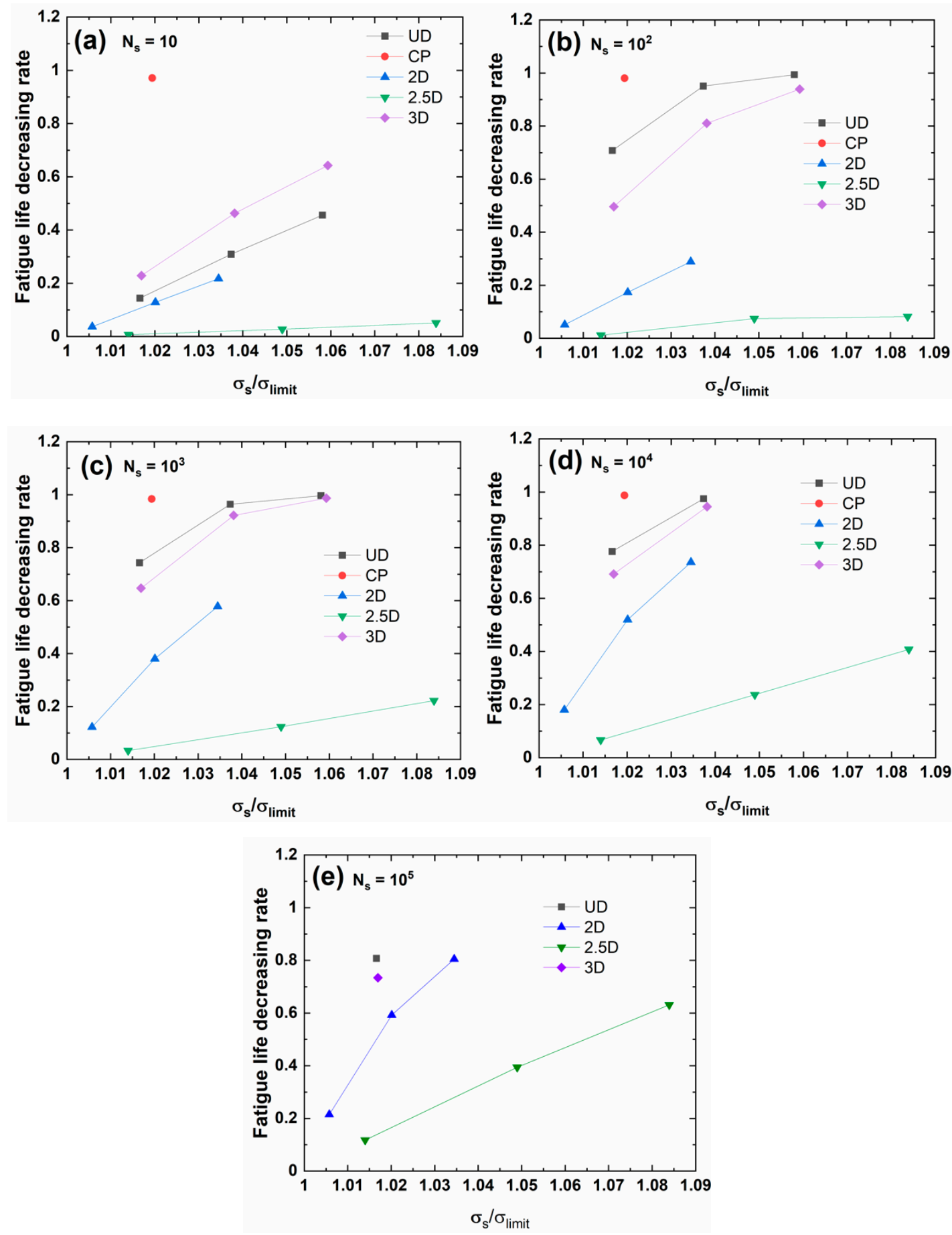

Figure 7. Fatigue life decreasing rate versus stochastic overloading stress for different occurrence applied cycle of (a) $N_{\mathrm{S}}=10 ;(\mathbf{b}) N_{\mathrm{S}}=10^{2} ;$ (c) $N_{\mathrm{S}}=10^{3} ;$ (d) $N_{\mathrm{S}}=10^{4} ;$ and, (e) $N_{\mathrm{s}}=10^{5}$.

For $N_{\mathrm{s}}=10,10^{2}, 10^{3}$, and $10^{4}$, under the same stochastic overloading stress level, the fatigue life decreasing rate is the highest for the cross-ply $\mathrm{C} / \mathrm{SiC}$ composite, which indicates that the fiber preform of the cross-ply is very sensitive to the stochastic overloading stress.

For $N_{\mathrm{s}}=10,10^{2}, 10^{3}, 10^{4}$, and $10^{5}$, under the same stochastic overloading stress level, the fatigue life decreasing rate is the lowest for the $2.5 \mathrm{D} \mathrm{C} / \mathrm{SiC}$ composite, which indicates that the fiber preform of 2.5D has high resistance to the stochastic overloading stress.

Among UD, 2D, and 3D C/SiC composites, at the initial stage of cyclic fatigue loading, i.e., $N_{\mathrm{s}}=$ 10 , under the same stochastic overloading stress, the fatigue life decrease rate of the $3 \mathrm{D} \mathrm{C} / \mathrm{SiC}$ is the 
highest; however, with the increasing applied cycle number, the fatigue life decreasing rate of the UD $\mathrm{C} / \mathrm{SiC}$ composite is the highest under the same stochastic overloading stress.

\section{Conclusions}

In this paper, a micromechanical fatigue limit stress model of fiber-reinforced CMCs subjected to stochastic overloading stress is developed. The fatigue limit stress for different $\mathrm{C} / \mathrm{SiC}$ composites is predicted. The relationships between fatigue life decreasing rate, stochastic overloading stress and corresponding occurrence cycle number, and broken fiber fraction are analyzed.

Under the same stochastic overloading stress level, the fatigue life decreasing rate increases with the stochastic overloading stress level and occurrence applied cycle of stochastic overloading for different fiber preforms. The broken fiber fraction increases when the stochastic overloading stress occurs, and the difference of the broken fiber fraction between the fatigue limit stress and stochastic overloading stress level increases with the applied cycle.

Under the same stochastic overloading stress level and occurrence applied cycle, the fatigue life decreasing rate is the highest for the cross-ply $\mathrm{C} / \mathrm{SiC}$ composite, and lowest for the $2.5 \mathrm{D} \mathrm{C} / \mathrm{SiC}$ composite.

For UD, CP, and 3D C/SiC composites, when the applied cycle is between $N_{\mathrm{s}}=10$ and $10^{2}$, the fatigue life decreasing rate increases rapidly with the occurrence applied cycle; however, when the applied cycle is higher than $N_{\mathrm{s}}=10^{2}$, the fatigue life decreasing rate increases slowly with the applied cycle.

For the $2 \mathrm{D}$ and $2.5 \mathrm{D} \mathrm{C} / \mathrm{SiC}$ composites, when the applied cycle is between $N_{\mathrm{s}}=10$ and $10^{2}$, the fatigue life decreasing rate increases slowly with the occurrence applied cycle; however, when the applied cycle is between $N_{\mathrm{s}}=10^{2}$ and $10^{5}$, the fatigue life decreasing rate increases rapidly with the applied cycle.

Funding: This research was funded by Fundamental Research Funds for the Central Universities, grant number NS2019038.

Acknowledgments: The author also wishes to thank two anonymous reviewers and editors for their helpful comments on an earlier version of the paper.

Conflicts of Interest: The author declares no conflict of interest.

\section{References}

1. Naslain, R. Design, Preparation and properties of non-oxide CMCs for application in engines and nuclear reactors: An overview. Compos. Sci. Technol. 2004, 64, 155-170. [CrossRef]

2. Wing, B.L.; Halloran, J.W. Subsurface oxidation of boron nitride coatings on silicon carbide fibers in $\mathrm{SiC} / \mathrm{SiC}$ ceramic matrix composites. Ceram. Int. 2018, 44, 17491-17505. [CrossRef]

3. Li, L.B. Time-Dependent Mechanical Behavior of Ceramic-Matrix Composites at Elevated Temperatures; Springer Nature Singapore Pte Ltd.: Singapore, 2020.

4. Li, L.B. Thermomechanical Fatigue of Ceramic-Matrix Composites; Wiley-VCH: Weinheim, Germany, 2019.

5. Reynaud, P. Cyclic fatigue of ceramic-matrix composites at ambient and elevated temperatures. Compos. Sci. Technol. 1996, 56, 809-814. [CrossRef]

6. Zhu, S.; Mizuno, M.; Kagawa, Y.; Mutoh, Y. Monotonic tension, fatigue and creep behavior of SiC-fiber-reinforced SiC-matrix composites: A review. Compos. Sci. Technol. 1999, 59, 833-851. [CrossRef]

7. Li, L.B. Tension-tension fatigue behavior of unidirectional C/SiC ceramic-matrix composite at room and elevated temperature and $800^{\circ} \mathrm{C}$ in air atmosphere. Materials 2015, 8, 3316-3333. [CrossRef]

8. Yang, F. Research on Fatigue Behavior of 2.5d Woven Ceramic Matrix Composites. Master's Thesis, Nanjing University of Aeronautics and Astronautics, Nanjing, China, December 2011.

9. Du, S.M.; Qiao, S.R.; Ji, G.C.; Han, D. Tension-tension fatigue behavior of 3D-C/SiC composite at room temperature and $1300{ }^{\circ} \mathrm{C}$. Mater. Eng. 2002, 9, 22-25.

10. Shuler, S.F.; Holmes, J.W.; Wu, X.; Roach, D. Influence of loading frequency on the room-temperature fatigue of a carbon-fiber/SiC-matrix composite. J. Am. Ceram. Soc. 1993, 76, 2327-2336. [CrossRef] 
11. Ruggles-Wrenn, M.B.; Christensen, D.T.; Chamberlain, A.L.; Lane, J.E.; Cook, T.S. Effect of frequency and environment on fatigue behavior of a CVI SiC/SiC ceramic matrix composite at $1200^{\circ} \mathrm{C}$. Compos. Sci. Technol. 2011, 71, 190-196. [CrossRef]

12. Li, L.B.; Reynaud, P.; Fantozzi, G. Cyclic-dependent damage evolution in self-healing woven SiC/[Si-B-C] ceramic-matrix composites at elevated temperatures. Materials 2020, 13, 1478. [CrossRef] [PubMed]

13. Li, L.B. Damage evolution and life prediction of cross-ply C/SiC ceramic-matrix composite under cyclic fatigue loading at room temperature and $800^{\circ} \mathrm{C}$ in air. Materials 2015, 8, 8539-8560. [CrossRef] [PubMed]

14. Ruggles-Wrenn, M.B.; Sharma, V. Effects of steam environment on fatigue behavior of two $\mathrm{SiC} /\left[\mathrm{SiC}_{+} \mathrm{Si}_{3} \mathrm{~N}_{4}\right]$ ceramic composites at $1300{ }^{\circ} \mathrm{C}$. Appl. Compos. Mater. 2011, 18, 385-396. [CrossRef]

15. Ruggles-Wrenn, M.B.; Lee, M.D. Fatigue behavior of an advanced SiC/SiC ceramic composite with a self-healing matrix at $1300{ }^{\circ} \mathrm{C}$ in air and in steam. Mater. Sci. Eng. A 2016, 677, 438-445. [CrossRef]

16. Ruggles-Wrenn, M.B.; Boucher, N.; Przybyla, C. Fatigue of three advanced SiC/SiC ceramic matrix composites at $1200{ }^{\circ} \mathrm{C}$ in air and in steam. Int. J. Appl. Ceram. Technol. 2018, 15, 3-15. [CrossRef]

17. Suzuki, T.; Mahfuz, H.; Takanashi, M. A new stiffness degradation model for fatigue life prediction of GFRPs under random loading. Int. J. Fatigue 2019, 119, 220-228. [CrossRef]

18. Li, L.B. Stress-rupture of fiber-reinforced ceramic-matrix composites with stochastic loading at intermediate temperatures. Part I: Theoretical analysis. Materials 2019, 12, 3123. [CrossRef] [PubMed]

19. Evans, A.G. Design and life prediction issues for high-temperature engineering ceramics and their composites. Acta Mater. 1997, 45, 23-40. [CrossRef]

20. Li, L.B. Fatigue life prediction of carbon fiber-reinforced ceramic-matrix composites at room and elevated temperatures. Part I: Experimental analysis. Appl. Compos. Mater. 2016, 23, 101-117. [CrossRef]

21. Li, L.B. Fatigue life prediction of carbon fiber-reinforced ceramic-matrix composites at room and elevated temperatures. Part II: Experimental comparisons. Appl. Compos. Mater. 2015, 22, 961-972.

22. Li, L.B. Fatigue life prediction of fiber-reinforced ceramic-matrix composites with different fiber preforms at room and elevated temperatures. Materials 2016, 9, 207. [CrossRef]

23. Curtin, W.A. Theory of mechanical properties of ceramic-matrix composites. J. Am. Ceram. Soc. 1991, 74, 2837-2845. [CrossRef]

(C) 2020 by the author. Licensee MDPI, Basel, Switzerland. This article is an open access article distributed under the terms and conditions of the Creative Commons Attribution (CC BY) license (http://creativecommons.org/licenses/by/4.0/). 